\title{
Differentiation Theory over Infinite-Dimensional Banach Spaces
}

\author{
Claudio Asci \\ Dipartimento di Matematica e Geoscienze, Università degli Studi di Trieste, Via Valerio 12/1, 34127 Trieste, Italy \\ Correspondence should be addressed to Claudio Asci; casci@units.it
}

Received 27 July 2016; Accepted 25 August 2016

Academic Editor: Tepper L Gill

Copyright (C) 2016 Claudio Asci. This is an open access article distributed under the Creative Commons Attribution License, which permits unrestricted use, distribution, and reproduction in any medium, provided the original work is properly cited.

\begin{abstract}
We study, for any positive integer $k$ and for any subset $I$ of $\mathbf{N}^{*}$, the Banach space $E_{I}$ of the bounded real sequences $\left\{x_{n}\right\}_{n \in I}$ and a measure over $\left(\mathbf{R}^{I}, \mathscr{B}^{(I)}\right)$ that generalizes the $k$-dimensional Lebesgue one. Moreover, we expose a differentiation theory for the functions defined over this space. The main result of our paper is a change of variables' formula for the integration of the measurable real functions on $\left(\mathbf{R}^{I}, \mathscr{B}^{(I)}\right)$. This change of variables is defined by some infinite-dimensional functions with properties that generalize the analogous ones of the standard finite-dimensional diffeomorphisms.
\end{abstract}

\section{Introduction}

The aim of this paper is to generalize the results of article [1], where, for any positive integer $k$ and for any subset $I$ of $\mathbf{N}^{*}$, we study a particular infinite-dimensional measure $\lambda_{N, a, v}^{(k, I)}$ that, in the case $I=\{1, \ldots, k\}$, coincides with the $k$-dimensional Lebesgue one on $\mathbf{R}^{k}$. The measure $\lambda_{N, a, v}^{(k, I)}$ is a product indexed by $I$ of $\sigma$-finite measures on the Borel $\sigma$-algebra $\mathscr{B}$ on $\mathbf{R}$ (by using a generalization of the Jessen theorem), and it is defined over the measurable space $\left(\mathbf{R}^{I}, \mathscr{B}^{(I)}\right)$, and in particular over $\left(E_{I}, \mathscr{B}_{I}\right)$, where $\mathscr{B}^{(I)}$ is the product indexed by $I$ of the same $\sigma$-algebra $\mathscr{B}, E_{I} \subset \mathbf{R}^{I}$ is the Banach space of the bounded real sequences $\left\{x_{n}\right\}_{n \in I}$, and $\mathscr{B}_{I}$ is the restriction to $E_{I}$ of $\mathscr{B}^{(I)}$.

In the mathematical literature, some articles introduced infinite-dimensional measures analogue of the Lebesgue one (see, e.g., the paper of Léandre [2], in the context of the noncommutative geometry, that one of Tsilevich et al. [3], which studies a family of $\sigma$-finite measures on $\mathbf{R}^{+}$, and that one of Baker [4], which defines a measure on $\mathbf{R}^{\mathbf{N}^{*}}$ that is not $\sigma$-finite).

In paper [1], the main result is a change of variables' formula for the integration of the measurable real functions on the space $\left(E_{I}, \mathscr{B}_{I}\right)$. This change of variables is defined by a particular class of linear functions over $E_{I}$, called $(m, \sigma)$ standard. A related problem is studied in the paper of Accardi et al. [5], where the authors describe the transformations of generalized measures on locally convex spaces under smooth transformations of these spaces.

In this paper, we prove that the change of variables' formula given in [1] can be extended by defining some infinite-dimensional functions with properties that generalize the analogous ones of the standard finite-dimensional diffeomorphisms.

In Section 2, we construct the infinite-dimensional Banach space $E_{I}$, and we define the continuous functions and the homeomorphisms over the open subsets of this space. Moreover, we recall some results about the integration of the measurable real functions defined on a measurable product space. In Section 3, we expose a differentiation theory in the infinite-dimensional context, and in particular we define the functions $C^{1}$ and the diffeomorphisms. Moreover, we introduce a class of functions, called $(m, \sigma)$-standard, that generalizes the set of the linear $(m, \sigma)$-standard functions given in [1], and we expose some properties of these functions. In Section 4, we present the main result of our paper, that is, a change of variables' formula for the integration of the measurable real functions on $\left(\mathbf{R}^{I}, \mathscr{B}^{(I)}\right)$; this change of variables is defined by the biunique, $C^{1}$ and $(m, \sigma)$ standard functions, with further properties (Theorem 47). This result agrees with the analogous finite-dimensional result. In Section 5, we expose some ideas for further study in the probability theory. 


\section{Construction of an Infinite-Dimensional Banach Space}

Henceforth, we will indicate by $\mathbf{N}^{*}$ and $\mathbf{R}^{*}$ the sets $\mathbf{N} \backslash$ $\{0\}$ and $\mathbf{R} \backslash\{0\}$, respectively. Let $I \neq \varnothing$ be a set and let $k \in \mathbf{N}^{*}$; indicate by $\tau$, by $\tau^{(k)}$, by $\tau^{(I)}$, by $\mathscr{B}$, by $\mathscr{B}^{(k)}$, by $\mathscr{B}^{(I)}$, by Leb, and by Leb ${ }^{(k)}$, respectively, the Euclidean topology on $\mathbf{R}$, the Euclidean topology on $\mathbf{R}^{k}$, the topology $\bigotimes_{i \in I} \tau$, the Borel $\sigma$-algebra on $\mathbf{R}$, the Borel $\sigma$-algebra on $\mathbf{R}^{k}$, the $\sigma$-algebra $\bigotimes_{i \in I} \mathscr{B}$, the Lebesgue measure on $\mathbf{R}$, and the Lebesgue measure on $\mathbf{R}^{k}$. Moreover, for any set $A \subset \mathbf{R}$, indicate by $\mathscr{B}(A)$ the $\sigma$-algebra induced by $\mathscr{B}$ on $A$, and by $\tau(A)$ the topology induced by $\tau$ on $A$; analogously, for any set $A \subset \mathbf{R}^{I}$, define the $\sigma$-algebra $\mathscr{B}^{(I)}(A)$ and the topology $\tau^{(I)}(A)$. Finally, if $S=\prod_{i \in I} S_{i}$ is a Cartesian product, for any $\left(x_{i}: i \in I\right) \in S$ and for any $\varnothing \neq H \subset I$, define $x_{H}=\left(x_{i}: i \in H\right) \in \prod_{i \in H} S_{i}$, and define the projection $\pi_{I, H}$ on $\prod_{i \in H} S_{i}$ as the function $\pi_{I, H}: S \rightarrow \prod_{i \in H} S_{i}$ given by $\pi_{I, H}\left(x_{I}\right)=x_{H}$.

Theorem 1. Let $I \neq \varnothing$ be a set and, for any $i \in I$, let $\left(S_{i}, \Sigma_{i}, \mu_{i}\right)$ be a measure space such that $\mu_{i}$ is finite. Moreover, suppose that, for some countable set $J \subset I, \mu_{i}$ is a probability measure for any $i \in I \backslash J$ and $\prod_{j \in J} \mu_{j}\left(S_{j}\right) \in \mathbf{R}^{+}$. Then, over the measurable space $\left(\prod_{i \in I} S_{i}, \bigotimes_{i \in I} \Sigma_{i}\right)$, there is a unique finite measure $\mu$, indicated by $\bigotimes_{i \in I} \mu_{i}$, such that, for any $H \subset I$ such that $|H|<+\infty$ and for any $A=\prod_{h \in H} A_{h} \times \prod_{i \in I \backslash H} S_{i} \in \bigotimes_{i \in I} \Sigma_{i}$, where $A_{h} \in$ $\Sigma_{h}, \forall h \in H$, we have $\mu(A)=\prod_{h \in H} \mu_{h}\left(A_{h}\right) \prod_{j \in J \backslash H} \mu_{j}\left(S_{j}\right)$. In particular, if $I$ is countable, then $\mu(A)=\prod_{i \in I} \mu_{i}\left(A_{i}\right)$ for any $A=\prod_{i \in I} A_{i} \in \bigotimes_{i \in I} \Sigma_{i}$.

Proof. See the proof of Corollary 4 in Asci [1].

Henceforth, we will suppose that $I, J$ are sets such that $\varnothing \neq I, J \subset \mathbf{N}^{*}$; moreover, for any $k \in \mathbf{N}^{*}$, we will indicate by $I_{k}=\left\{i_{1}, \ldots, i_{k}\right\}$ the set of the first $k$ elements of $I$ (with the natural order and with the convention $I_{k}=I$ if $|I|<k$ ) and analogously $J_{k}$; finally, for any $j=i_{n} \in I$, set $|j|=n$.

Theorem 2. Let $\left(S_{i}, \Sigma_{i}, \mu_{i}\right)$ be a probability space, for any $i \in I$, let $(S, \Sigma, \mu)=\left(\prod_{i \in I} S_{i}, \bigotimes_{i \in I} \Sigma_{i}, \bigotimes_{i \in I} \mu_{i}\right)$, and let $f \in$ $L^{1}(S, \Sigma, \mu)$. Moreover, for any $H \subset I$ such that $\varnothing \neq|H|<+\infty$, define the measurable function $f_{H^{c}}:(S, \Sigma) \rightarrow(\mathbf{R}, \mathscr{B})$ by

$$
f_{H^{c}}(x)=\int_{S_{H}} f\left(x_{H}, x_{H^{c}}\right) d \mu_{H}\left(x_{H}\right),
$$

where $\left(S_{H}, \Sigma_{H}, \mu_{H}\right)$ is the probability space $\left(\prod_{i \in H} S_{i}, \bigotimes_{i \in H} \Sigma_{i}, \bigotimes_{i \in H} \mu_{i}\right)$. Then, $\mu$-a.e. one has $\lim _{n \rightarrow+\infty} f_{I_{n}^{c}}=\int_{S} f d \mu$.

Proof. See, for example, Theorem 3, page 349, in Rao [6].

Corollary 3. Let $\left(S_{i}, \Sigma_{i}, \mu_{i}\right)$ be a measure space such that $\mu_{i}$ is finite, for any $i \in I$, and $\prod_{i \in I} \mu_{i}\left(S_{i}\right) \in[0,+\infty)$; moreover, let $(S, \Sigma, \mu)=\left(\prod_{i \in I} S_{i}, \bigotimes_{i \in I} \Sigma_{i}, \bigotimes_{i \in I} \mu_{i}\right)$, let $f \in L^{1}(S, \Sigma, \mu)$, and let the measurable function $f_{I_{n}^{c}}:(S, \Sigma) \rightarrow(\mathbf{R}, \mathscr{B})$ defined by (1), for any $n \in \mathbf{N}^{*}$. Then, $\mu$-a.e. one has $\lim _{n \rightarrow+\infty} f_{I_{n}^{c}}=$ $\int_{S} f d \mu$.
Proof. Suppose that $\mu_{i}\left(S_{i}\right) \neq 0, \forall i \in I$; then, $\bar{\mu}_{i}=\mu_{i} / \mu_{i}\left(S_{i}\right)$ is a probability measure, and also $\bar{\mu}=\bigotimes_{i \in I} \bar{\mu}_{i}, \bar{\mu}_{I_{n}}=\bigotimes_{i \in I_{n}} \bar{\mu}_{i}$, and $\bar{\mu}_{I_{n}^{c}}=\bigotimes_{i \in I_{n}^{c}} \bar{\mu}_{i}, \forall n \in \mathbf{N}^{*}$; then, define the function $\bar{f}_{I_{n}^{c}}$ : $(S, \Sigma) \rightarrow(\mathbf{R}, \mathscr{B})$ by

$$
\bar{f}_{I_{n}^{c}}(x)=\int_{S_{I_{n}}} f\left(x_{I_{n}}, x_{I_{n}^{c}}\right) d \bar{\mu}_{I_{n}}\left(x_{I_{n}}\right) .
$$

From Theorem $2, \bar{\mu}$-a.e., we have

$$
\begin{aligned}
\lim _{n \rightarrow+\infty} f_{I_{n}^{c}} & =\lim _{n \rightarrow+\infty}\left(\prod_{i \in I_{n}} \mu_{i}\left(S_{i}\right)\right) \bar{f}_{I_{n}^{c}} \\
& =\prod_{i \in I} \mu_{i}\left(S_{i}\right) \int_{S} f d \bar{\mu}=\int_{S} f d \mu .
\end{aligned}
$$

Conversely, if $\mu_{i}\left(S_{i}\right)=0$, for some $i \in I$, then

$$
\lim _{n \rightarrow+\infty} f_{I_{n}^{c}}=0=\int_{S} f d \mu .
$$

Thus, since $\mu=\left(\prod_{i \in I} \mu_{i}\left(S_{i}\right)\right) \bar{\mu}$, we obtain the statement.

Definition 4. For any set $I \neq \varnothing$, define the function $\|\cdot\|_{I}$ : $\mathbf{R}^{I} \rightarrow[0,+\infty]$ by

$$
\|x\|_{I}=\sup _{i \in I}\left|x_{i}\right|, \quad \forall x=\left(x_{i}: i \in I\right) \in \mathbf{R}^{I},
$$

and define the vector space

$$
E_{I}=\left\{x \in \mathbf{R}^{I}:\|x\|_{I}<+\infty\right\} .
$$

Moreover, indicate by $\mathscr{B}_{I}$ the $\sigma$-algebra $\mathscr{B}^{(I)}\left(E_{I}\right)$, by $\tau_{I}$ the topology $\tau^{(I)}\left(E_{I}\right)$ induced on $E_{I}$ by the product topology $\tau^{(I)}\left(\mathbf{R}^{I}\right)$, and by $\tau_{\|\cdot\|_{I}}$ the topology induced on $E_{I}$ by the distance $d: E_{I} \times E_{I} \rightarrow[0,+\infty)$ defined by $d(x, y)=\|x-y\|_{I}$, $\forall x, y \in E_{I}$; furthermore, for any set $A \subset E_{I}$, indicate by $\tau_{\|\cdot\|_{I}}(A)$ the topology induced by $\tau_{\|\cdot\|_{I}}$ on $A$. Finally, for any $x_{0} \in E_{I}$ and for any $\delta>0$, indicate by $B_{I}\left(x_{0}, \delta\right)$ the set $\left\{x \in E_{I}:\left\|x-x_{0}\right\|_{I}<\delta\right\}$.

Observe that $\tau^{(I)}(A) \subset \tau_{\|\cdot\|_{I}}(A), \forall A \subset E_{I}$; moreover, $E_{I}$ is a Banach space, with the norm $\|\cdot\|_{I}$ (see, e.g., the proof of Remark 2 in [1]).

Proposition 5. Let $H$ and $I$ be sets such that $\varnothing \neq H \subset I$; then, the function $\pi_{I, H}:\left(\mathbf{R}^{I}, \tau^{(I)}\right) \rightarrow\left(\mathbf{R}^{H}, \tau^{(H)}\right)$ is continuous and open.

Proof. See, for example, the theory of the product spaces in Weidmann's book [7].

Proposition 6. Let $H$ and $I$ be sets such that $\varnothing \neq H \subset I$, and let $\bar{\pi}_{I, H}: E_{I} \rightarrow E_{H}$ be the function given by $\bar{\pi}_{I, H}(x)=\pi_{I, H}(x)$, for any $x \in E_{I}$; then,

(1) $\bar{\pi}_{I, H}:\left(E_{I}, \tau_{I}\right) \rightarrow\left(E_{H}, \tau_{H}\right)$ is continuous and open;

(2) $\bar{\pi}_{I, H}:\left(E_{I}, \tau_{\|\cdot\|_{I}}\right) \rightarrow\left(E_{H}, \tau_{\|\cdot\|_{H}}\right)$ is continuous and open. 
Proof of (1). $\forall A \in \tau_{H}$, there exists $B \in \tau^{(H)}$ such that $A=$ $B \cap E_{H}$, and so $\bar{\pi}_{I, H}^{-1}(A)=\left(\pi_{I, H}^{-1}\left(B \cap E_{H}\right)\right) \cap E_{I}=\pi_{I, H}^{-1}(B) \cap E_{I}$; then, since $\pi_{I, H}^{-1}(B) \in \tau^{(I)}$ by Proposition 5 , we have $\bar{\pi}_{I, H}^{-1}(A) \epsilon$ $\tau_{I}$, and so $\bar{\pi}_{I, H}$ is a continuous function. Moreover, $\forall A \in \tau_{I}$, there exists $B \in \tau^{(I)}$ such that $A=B \cap E_{I}$, and so $\bar{\pi}_{I, H}(A)=$ $\pi_{I, H}\left(B \cap E_{I}\right)=\pi_{I, H}(B) \cap E_{H}$; then, since $\pi_{I, H}(B) \in \tau^{(H)}$ by Proposition 5 , we have $\bar{\pi}_{I, H}(A) \in \tau_{H}$, and so $\bar{\pi}_{I, H}$ is an open function.

Proof of (2). $\forall A \in \tau_{\|\cdot\|_{H}}$ and $\forall x=\left(x_{i}: i \in I\right) \in \bar{\pi}_{I, H}^{-1}(A)$, we have $\left(x_{i}: i \in H\right) \in A$, and so there exists $a \in \mathbf{R}^{+}$and $\left(y_{i}: i \in\right.$ $H) \in A$ such that $\left(x_{i}: i \in H\right) \in \prod_{i \in H}\left(y_{i}-a, y_{i}+a\right) \subset A$; let $z=\left(z_{i}: i \in I\right) \in \bar{\pi}_{I, H}^{-1}(A)$ such that $z_{i}=x_{i}, \forall i \in I \backslash H$, and $z_{i}=y_{i}, \forall i \in H$; then, $x \in \prod_{i \in I}\left(z_{i}-a, z_{i}+a\right) \subset \bar{\pi}_{I, H}^{-1}(A)$, and so $\bar{\pi}_{I, H}$ is a continuous function. Moreover, $\forall A \in \tau_{\|\cdot\|_{I}}$ and $\forall x=\left(x_{i}: i \in H\right) \in \bar{\pi}_{I, H}(A)$, let $y=\left(y_{i}: i \in I\right) \in A$ such that $y_{i}=x_{i}, \forall i \in H$; since $A \in \tau_{\|\cdot\|_{I}}$, there exists $a \in \mathbf{R}^{+}$and $\left(z_{i}: i \in I\right) \in A$ such that $y \in \prod_{i \in I}\left(z_{i}-a, z_{i}+a\right) \subset A$, and so $x \in \prod_{i \in H}\left(z_{i}-a, z_{i}+a\right) \subset \bar{\pi}_{I, H}(A)$; then, $\bar{\pi}_{I, H}$ is an open function.

Definition 7. Let $U \in \tau_{\|\cdot\|_{J}}$, let $x_{0} \in U$, let $l \in E_{I}$, and let $\varphi: U \subset E_{J} \rightarrow E_{I}$ be a function; we say that $\lim _{x \rightarrow x_{0}} \varphi(x)=l$ if, for any neighbourhood $M \in \tau_{\|\cdot\|_{I}}$ of $\varphi\left(x_{0}\right)$, there exists a neighbourhood $N \in \tau_{\|\cdot\|_{J}}(U)$ of $x_{0}$ such that, for any $x \in N \backslash$ $\left\{x_{0}\right\}$, we have $\varphi(x) \in M$.

Definition 8. Let $U \in \tau_{\|\cdot\|_{J}}$ and let $\varphi: U \subset E_{J} \rightarrow E_{I}$ be a function; we say that $\varphi$ is continuous in $x_{0} \in U$ if $\lim _{x \rightarrow x_{0}} \varphi(x)=\varphi\left(x_{0}\right)$, and we say that $\varphi$ is continuous in $U$ if $\varphi$ is continuous in $x_{0}$ for any $x_{0} \in U$.

Remark 9. Let $U \in \tau_{\|\cdot\|_{I}}$, let $V \in \tau_{\|\cdot\|_{I}}$, and let $\varphi: U \subset E_{J} \rightarrow$ $V \subset E_{I}$ be a function; then, $\varphi:\left(U, \tau_{\|\cdot\|_{J}}(U)\right) \rightarrow\left(V, \tau_{\|\cdot\|_{I}}(V)\right)$ is continuous if and only if $\varphi$ is continuous in $U$.

Remark 10. Let $H, I$, and $J$ be sets such that $\varnothing \neq H \subset I$, let $U \in \tau_{\|\cdot\|_{J}}$, and let $\varphi: U \subset E_{J} \rightarrow E_{I}$ be a function continuous in $U$; then, the function $\left(\pi_{I, H} \circ \varphi\right): U \rightarrow E_{H}$ is continuous in $U$.

Proof. The statement follows from Proposition 6.
Definition 11. Let $U \in \tau_{\|\cdot\|_{J}}$ and let $V \in \tau_{\|\cdot\|_{I}}$; a function $\varphi$ : $U \subset E_{J} \rightarrow V \subset E_{I}$ is called homeomorphism if $\varphi$ is biunique and the functions $\varphi:\left(U, \tau_{\|\cdot\|_{I}}(U)\right) \rightarrow\left(V, \tau_{\|\cdot\|_{I}}(V)\right)$ and $\varphi^{-1}:$ $\left(V, \tau_{\|\cdot\|_{I}}(V)\right) \rightarrow\left(U, \tau_{\|\cdot\|_{J}}(U)\right)$ are continuous.

\section{Differentiation Theory in the Infinite-Dimensional Context}

The following concept generalizes Definition 6 in [1] (see also the theory in the Lang's book [8]).

Definition 12. Let $A=\left(a_{i j}\right)_{i \in I, j \in J}$ be a real matrix $I \times J$ (eventually infinite); then, define the linear function $A=$ $\left(a_{i j}\right)_{i \in I, j \in J}: E_{J} \rightarrow \mathbf{R}^{I}$, and write $x \rightarrow A x$, in the following manner:

$$
(A x)_{i}=\sum_{j \in J} a_{i j} x_{j}, \quad \forall x \in E_{J}, \forall i \in I,
$$

on condition that, for any $i \in I$, the sum in (7) converges to a real number.

Definition 13. Let $U \in \tau_{\|\cdot\|_{J}}$; a function $\varphi: U \subset E_{J} \rightarrow E_{I}$ is called differentiable in $x_{0} \in U$ if there exists a linear and continuous function $A: E_{J} \rightarrow E_{I}$ defined by a real matrix $A=\left(a_{i j}\right)_{i \in I, j \in J}$, and one has

$$
\lim _{h \rightarrow 0} \frac{\left\|\varphi\left(x_{0}+h\right)-\varphi\left(x_{0}\right)-A h\right\|_{I}}{\|h\|_{J}}=0 .
$$

If $\varphi$ is differentiable in $x_{0}$ for any $x_{0} \in U, \varphi$ is called differentiable in $U$. The function $A$ is called differential of the function $\varphi$ in $x_{0}$, and it is indicated by the symbol $d \varphi\left(x_{0}\right)$.

Remark 14. Let $U \in \tau_{\|\cdot\|_{J}}$ and let $\varphi, \psi: U \subset E_{J} \rightarrow E_{I}$ be differentiable functions in $x_{0} \in U$; then, for any $\alpha, \beta \in \mathbf{R}$, the function $\alpha \varphi+\beta \psi$ is differentiable in $x_{0}$, and $d(\alpha \varphi+\beta \psi)\left(x_{0}\right)=$ $\alpha d \varphi\left(x_{0}\right)+\beta d \psi\left(x_{0}\right)$.

Proof. Observe that

$$
\begin{aligned}
& \lim _{h \rightarrow 0} \frac{\left\|(\alpha \varphi+\beta \psi)\left(x_{0}+h\right)-(\alpha \varphi+\beta \psi)\left(x_{0}\right)-\left(\alpha d \varphi\left(x_{0}\right)+\beta d \psi\left(x_{0}\right)\right) h\right\|_{I}}{\|h\|_{J}} \\
& \quad=\lim _{h \rightarrow 0} \frac{\left\|\alpha \varphi\left(x_{0}+h\right)-\alpha \varphi\left(x_{0}\right)-\alpha d \varphi\left(x_{0}\right) h+\beta \psi\left(x_{0}+h\right)-\beta \psi\left(x_{0}\right)-\beta d \psi\left(x_{0}\right) h\right\|_{I}}{\|h\|_{J}} \\
& \quad \leq \lim _{h \rightarrow 0} \frac{\left\|\alpha \varphi\left(x_{0}+h\right)-\alpha \varphi\left(x_{0}\right)-\alpha d \varphi\left(x_{0}\right) h\right\|_{I}}{\|h\|_{J}}+\lim _{h \rightarrow 0} \frac{\left\|\beta \psi\left(x_{0}+h\right)-\beta \psi\left(x_{0}\right)-\beta d \psi\left(x_{0}\right) h\right\|_{I}}{\|h\|_{J}}=0 \Longrightarrow \\
& d(\alpha \varphi+\beta \psi)\left(x_{0}\right)=\alpha d \varphi\left(x_{0}\right)+\beta d \psi\left(x_{0}\right) .
\end{aligned}
$$


Remark 15. A linear and continuous function $A=\left(a_{i j}\right)_{i \in I, j \in J}$ : $E_{J} \rightarrow E_{I}$, defined by

$$
(A x)_{i}=\sum_{j \in J} a_{i j} x_{j}, \quad \forall x \in E_{J}, \quad \forall i \in I,
$$

is differentiable and $d \varphi\left(x_{0}\right)=A$, for any $x_{0} \in E_{J}$.

Remark 16. Let $U \in \tau_{\|\cdot\|_{I}}$ and let $\varphi: U \subset E_{J} \rightarrow E_{I}$ be a function differentiable in $x_{0} \in U$; then, for any $i \in I$, the component $\varphi_{i}: U \rightarrow \mathbf{R}$ is differentiable in $x_{0}$, and $d \varphi_{i}\left(x_{0}\right)$ is the matrix $A_{i}$ given by the $i$ th row of $A=d \varphi\left(x_{0}\right)$. Moreover, if $|I|<+\infty$ and $\varphi_{i}: U \subset E_{J} \rightarrow \mathbf{R}$ is differentiable in $x_{0}$, for any $i \in I$, then $\varphi: U \subset E_{J} \rightarrow E_{I}$ is differentiable in $x_{0}$.

Remark 17. Let $U \in \tau_{\|\cdot\|_{I}}$ and let $\varphi: U \subset E_{J} \rightarrow E_{I}$ be a function differentiable in $x_{0} \in U$; then, $\varphi$ is continuous in $x_{0}$.

Proof. $\forall x \in U$, set

$$
\sigma\left(x, x_{0}\right)=f(x)-f\left(x_{0}\right)-d f\left(x_{0}\right)\left(x-x_{0}\right) .
$$

From (8), we have $\lim _{x \rightarrow x_{0}}\left\|\sigma\left(x, x_{0}\right)\right\|_{I}=0$; moreover,

$$
\begin{aligned}
\left\|f(x)-f\left(x_{0}\right)\right\|_{I}= & \left\|d f\left(x_{0}\right)\left(x-x_{0}\right)+\sigma\left(x, x_{0}\right)\right\|_{I} \\
\leq & \left\|d f\left(x_{0}\right)\right\|\left\|x-x_{0}\right\|_{J} \\
& +\left\|\sigma\left(x, x_{0}\right)\right\|_{I},
\end{aligned}
$$

from which $\lim _{x \rightarrow x_{0}} f(x)=f\left(x_{0}\right)$.

Definition 18. Let $U \in \tau_{\|\cdot\|_{J}}$ and let $v \in E_{J}$ such that $\|v\|_{J}=1$; a function $\varphi: U \subset E_{J} \rightarrow \mathbf{R}$ is called derivable in $x_{0} \in U$ in the direction $v$ if there exists the limit

$$
\lim _{t \rightarrow 0} \frac{\varphi\left(x_{0}+t v\right)-\varphi\left(x_{0}\right)}{t} .
$$

This limit is indicated by $(\partial \varphi / \partial v)\left(x_{0}\right)$, and it is called derivative of $\varphi$ in $x_{0}$ in the direction $v$. If for some $j \in J$ one has $v=e_{j}$, where $\left(e_{j}\right)_{k}=\delta_{j k}$, for any $k \in J$, indicate $(\partial \varphi / \partial v)\left(x_{0}\right)$ by $\left(\partial \varphi / \partial x_{j}\right)\left(x_{0}\right)$, and call it partial derivative of $\varphi$ in $x_{0}$, with respect to $x_{j}$. Moreover, if there exists the linear function defined by the matrix $J_{\varphi}\left(x_{0}\right)$, where $\left(J_{\varphi}\left(x_{0}\right)\right)_{i j}=$ $\left(\partial \varphi_{i} / \partial x_{j}\right)\left(x_{0}\right)$, for any $i \in I$ and $j \in J$, then $J_{\varphi}\left(x_{0}\right)$ is called Jacobian matrix of the function $\varphi$ in $x_{0}$.

Remark 19. Let $U \in \tau_{\|\cdot\|_{J}}$ and suppose that a function $\varphi: U \subset$ $E_{J} \rightarrow E_{I}$ is differentiable in $x_{0} \in U$; then, for any $v \in E_{J}$ such that $\|v\|_{J}=1$ and for any $i \in I$, the function $\varphi_{i}: U \subset E_{J} \rightarrow \mathbf{R}$ is derivable in $x_{0}$ in the direction $v$, and one has

$$
\frac{\partial \varphi_{i}}{\partial v}\left(x_{0}\right)=d \varphi_{i}\left(x_{0}\right) v
$$

Proof. $\forall h \in E_{J}$, by setting $h=t v$, for some matrix $A=$ $\left(a_{i j}\right)_{i \in I, j \in J}$ we have

$$
\begin{aligned}
\lim _{t \rightarrow 0} & \frac{\left|\varphi_{i}\left(x_{0}+t v\right)-\varphi_{i}\left(x_{0}\right)-A_{i}(t v)\right|}{|t|} \\
= & \lim _{h \rightarrow 0} \frac{\left|\varphi_{i}\left(x_{0}+h\right)-\varphi_{i}\left(x_{0}\right)-A_{i} h\right|}{\|h\|_{J}}=0 .
\end{aligned}
$$

Then, since $A_{i}(t v)=t A_{i} v$, we have

$$
\lim _{t \rightarrow 0} \frac{\varphi_{i}\left(x_{0}+t v\right)-\varphi_{i}\left(x_{0}\right)}{t}=A_{i} v,
$$

from which

$$
\frac{\partial \varphi_{i}}{\partial v}\left(x_{0}\right)=A_{i} v=d \varphi_{i}\left(x_{0}\right) v
$$

Corollary 20. Let $U \in \tau_{\|\cdot\|_{J}}$ and let $\varphi: U \subset E_{J} \rightarrow E_{I}$ be a function differentiable in $x_{0} \in U$; then, the linear function $J_{\varphi}\left(x_{0}\right): E_{J} \rightarrow E_{I}$ is defined and continuous; moreover, for any $h \in E_{J}$, one has $d \varphi\left(x_{0}\right)(h)=J_{\varphi}\left(x_{0}\right) h$.

Proof. From Remark 19, $\forall i \in I$ and $\forall j \in J$, we have $\left(d \varphi\left(x_{0}\right)\right)_{i j}=d \varphi_{i}\left(x_{0}\right)\left(e_{j}\right)=\left(\partial \varphi_{i} / \partial x_{j}\right)\left(x_{0}\right)=\left(J_{\varphi}\left(x_{0}\right)\right)_{i j}$.

Theorem 21. Let $U \in \tau_{\|\cdot\|_{I}}$, let $\varphi: U \subset E_{J} \rightarrow E_{I}$ be a function differentiable in $x_{0} \in U$, let $V \in \tau_{\|\cdot\|_{I}}$ such that $V \supset \varphi(U)$, and let $\psi: V \subset E_{I} \rightarrow E_{H}$ be a function differentiable in $y_{0}=\varphi\left(x_{0}\right)$. Then, the function $\psi \circ \varphi$ is differentiable in $x_{0}$, and one has $d(\psi \circ \varphi)\left(x_{0}\right)=d \psi\left(y_{0}\right) \circ d \varphi\left(x_{0}\right)$.

Proof. The proof is analogous to that one true in the particular case $|H|<+\infty,|I|<+\infty,|J|<+\infty$ (see, e.g., the Lang's book [9]).

Definition 22. Let $U \in \tau_{\|\cdot\|_{J}}$, let $i, j \in J$, and let $\varphi: U \subset$ $E_{J} \rightarrow \mathbf{R}$ be a function derivable in $x_{0} \in U$ with respect to $x_{i}$, such that the function $\partial \varphi / \partial x_{i}$ is derivable in $x_{0}$ with respect to $x_{j}$. Indicate $\left(\partial / \partial x_{j}\right)\left(\partial \varphi / \partial x_{i}\right)\left(x_{0}\right)$ by $\left(\partial^{2} \varphi / \partial x_{j} \partial x_{i}\right)\left(x_{0}\right)$ and call it second partial derivative of $\varphi$ in $x_{0}$ with respect to $x_{i}$ and $x_{j}$. If $i=j$, it is indicated by $\left(\partial^{2} \varphi / \partial x_{i}^{2}\right)\left(x_{0}\right)$. Analogously, for any $k \in \mathbf{N}^{*}$ and for any $j_{1}, \ldots, j_{k} \in J$, define $\left(\partial^{k} \varphi /\left(\partial x_{j_{k}} \cdots \partial x_{j_{1}}\right)\right)\left(x_{0}\right)$ and call it $k$ th partial derivative of $\varphi$ in $x_{0}$ with respect to $x_{j_{1}}, \ldots, x_{j_{k}}$.

Definition 23. Let $U \in \tau_{\|\cdot\|_{J}}$ and let $k \in \mathbf{N}^{*}$; a function $\varphi$ : $U \subset E_{J} \rightarrow E_{I}$ is called $C^{k}$ in $x_{0} \in U$ if, in a neighbourhood $V \in \tau_{\|\cdot\|_{J}}(U)$ of $x_{0}$, for any $i \in I$ and for any $j_{1}, \ldots, j_{k} \in J$, there exists the function defined by $x \rightarrow\left(\partial^{k} \varphi_{i} /\left(\partial x_{j_{k}} \cdots \partial x_{j_{1}}\right)\right)(x)$, and this function is continuous in $x_{0} ; \varphi$ is called $C^{k}$ in $U$ if, for any $x_{0} \in U, \varphi$ is $C^{k}$ in $x_{0}$. Moreover, $\varphi$ is called strongly $C^{1}$ in $x_{0} \in U$ if, in a neighbourhood $V \in \tau_{\|\cdot\|_{J}}(U)$ of $x_{0}$, there exists the function defined by $x \rightarrow J_{\varphi}(x)$, and this function is continuous in $x_{0}$, with $\left\|J_{\varphi}\left(x_{0}\right)\right\|<+\infty$. Finally, $\varphi$ is called strongly $C^{1}$ in $U$ if, for any $x_{0} \in U, \varphi$ is strongly $C^{1}$ in $x_{0}$.

Definition 24. Let $U \in \tau_{\|\cdot\|_{J}}$ and let $V \in \tau_{\|\cdot\|_{I}}$; a function $\varphi$ : $U \subset E_{J} \rightarrow V \subset E_{I}$ is called diffeomorphism if $\varphi$ is biunique and $C^{1}$ in $U$, and the function $\varphi^{-1}: V \subset E_{I} \rightarrow U \subset E_{J}$ is $C^{1}$ in $V$.

Remark 25. Let $U \in \tau_{\|\cdot\|_{J}}$ and let $\varphi: U \subset E_{J} \rightarrow E_{I}$ be a function $C^{1}$ in $x_{0} \in U$, where $|I|<+\infty,|J|<+\infty$; then, $\varphi$ is strongly $C^{1}$ in $x_{0}$. 
Theorem 26. Let $U \in \tau_{\|\cdot\|_{J}}$, let $\varphi: U \subset E_{J} \rightarrow \mathbf{R}$ be a function $C^{k}$ in $x_{0} \in U$, let $i_{1}, \ldots, i_{k} \in J$, and let $j_{1}, \ldots, j_{k} \in J$ be a permutation of $i_{1}, \ldots, i_{k}$. Then, one has

$$
\frac{\partial^{k} \varphi}{\partial x_{i_{1}} \cdots \partial x_{i_{k}}}\left(x_{0}\right)=\frac{\partial^{k} \varphi}{\partial x_{j_{1}} \cdots \partial x_{j_{k}}}\left(x_{0}\right) .
$$

Proof. The proof is analogous to that one true in the particular case $|J|<+\infty$ (see, e.g., the Lang's book [9]).

Proposition 27. Let $U=\left(\prod_{j \in J} U_{j}\right) \cap E_{J} \in \tau_{\|\cdot\|_{J}}$, where $U_{j} \in \tau$, for any $j \in J$, and let $\varphi: U \subset E_{J} \rightarrow E_{I}$ be a function $C^{1}$ in $x_{0} \in U$, such that

$$
\varphi_{i}(x)=\sum_{j \in J} \varphi_{i j}\left(x_{j}\right), \quad \forall x=\left(x_{j}: j \in J\right) \in U, \forall i \in I,
$$

where $\varphi_{i j}: U_{j} \rightarrow \mathbf{R}, \forall i \in I$ and $\forall j \in J$; moreover, suppose that there exists a neighbourhood $V \in \tau_{\|\cdot\|_{I}}(U)$ of $x_{0}$ such that $\sup _{x \in V}\left\|J_{\varphi}(x)\right\|<+\infty$. Then, $\varphi$ is continuous in $x_{0}$; in particular, if $\varphi$ is strongly $C^{1}$ in $x_{0}$ and $|I|<+\infty, \varphi$ is differentiable in $x_{0}$.

Proof. Since $\varphi$ is $C^{1}$ in $x_{0}$, from (19) there exists a neighbourhood of $x_{0}=\left(x_{0 j}: j \in J\right)$ that we can suppose to be $V$, such that $V=\prod_{j \in J} V_{j} \subset U$, where $V_{j}=\left(x_{0 j}-\gamma_{j}, x_{0 j}+\delta_{j}\right)$, $\forall j \in J$, and such that, $\forall i \in I$ and $\forall j \in J$, $\varphi_{i j}^{\prime}$ exists in $V_{j}$. Let $x=\left(x_{j}: j \in J\right) \in V$; from the Lagrange theorem, $\forall i \in I$ and $\forall j \in J$, there exists $\xi_{i j} \in\left(\min \left\{x_{0 j}, x_{j}\right\}, \max \left\{x_{0 j}, x_{j}\right\}\right) \subset V_{j}$ such that $\varphi_{i j}\left(x_{j}\right)-\varphi_{i j}\left(x_{0 j}\right)=\varphi_{i j}^{\prime}\left(\xi_{i j}\right)\left(x_{j}-x_{0 j}\right)$. Then,

$$
\begin{aligned}
\varphi_{i}(x)-\varphi_{i}\left(x_{0}\right) & =\sum_{j \in J} \varphi_{i j}^{\prime}\left(\xi_{i j}\right)\left(x_{j}-x_{0 j}\right) \Longrightarrow \\
\varphi(x)-\varphi\left(x_{0}\right) & =A_{\varphi}\left(x, x_{0}\right)\left(x-x_{0}\right),
\end{aligned}
$$

where $A_{\varphi}\left(x, x_{0}\right)=\left(\varphi_{i j}^{\prime}\left(\xi_{i j}\right)\right)_{i \in I, j \in J}$. Thus, if $\sup _{x \in V}\left\|J_{\varphi}(x)\right\|=$ $c<+\infty, \forall x \in V$, we have

$$
\begin{aligned}
\left\|A_{\varphi}\left(x, x_{0}\right)\right\| & =\sup _{i \in I}\left\|\left(A_{\varphi}\left(x, x_{0}\right)\right)_{i}\right\| \\
& \leq \sup _{i \in I}\left\|J_{\varphi}\left(\xi_{i j}: j \in J\right)\right\| \leq c,
\end{aligned}
$$

from which

$$
\left\|\varphi(x)-\varphi\left(x_{0}\right)\right\|_{I} \leq c\left\|x-x_{0}\right\|_{J}
$$

then, $\varphi$ is continuous in $x_{0}$. Moreover, we have

$$
\begin{aligned}
& \varphi(x)-\varphi\left(x_{0}\right)-J_{\varphi}\left(x_{0}\right)\left(x-x_{0}\right) \\
& =\left(A_{\varphi}\left(x, x_{0}\right)-J_{\varphi}\left(x_{0}\right)\right)\left(x-x_{0}\right) \Longrightarrow \\
& \frac{\left\|\varphi(x)-\varphi\left(x_{0}\right)-J_{\varphi}\left(x_{0}\right)\left(x-x_{0}\right)\right\|_{I}}{\left\|x-x_{0}\right\|_{J}} \\
& \leq\left\|A_{\varphi}\left(x, x_{0}\right)-J_{\varphi}\left(x_{0}\right)\right\| \\
& \leq \sup _{i \in I}\left\|J_{\varphi}\left(\xi_{i j}: j \in J\right)-J_{\varphi}\left(x_{0}\right)\right\| .
\end{aligned}
$$

Then, if $\varphi$ is strongly $C^{1}$ in $x_{0}$ and $|I|<+\infty$, we obtain

$$
\lim _{x \rightarrow x_{0}} \frac{\left\|\varphi(x)-\varphi\left(x_{0}\right)-J_{\varphi}\left(x_{0}\right)\left(x-x_{0}\right)\right\|_{I}}{\left\|x-x_{0}\right\|_{J}}=0,
$$

and so $\varphi$ is differentiable in $x_{0}$, with $d \varphi\left(x_{0}\right)=J_{\varphi}\left(x_{0}\right)$.

Definition 28. Let $m \in \mathbf{N}^{*}$, let $U=\left(U^{(m)} \times \prod_{j \in I \backslash I_{m}} A_{j}\right) \cap E_{I} \in$ $\tau_{\|\cdot\|_{I}}$, where $U^{(m)} \in \tau^{(m)}, A_{j} \subset \mathbf{R}$ is an open interval, for any $j \in I \backslash I_{m}$, and let $\sigma: I \backslash I_{m} \rightarrow I \backslash I_{m}$ be an increasing function. A function $\varphi: U \subset E_{I} \rightarrow E_{I}$ is called $(m, \sigma)$-standard if

(1) $\forall i \in I$, there exist some functions $\varphi_{i}^{(m, m)}: U^{(m)} \rightarrow \mathbf{R}$ and $\left\{\varphi_{i j}\right\}_{j \in I \backslash I_{m}}: A_{j} \rightarrow \mathbf{R}$ such that, $\forall x \in U$, one has $\varphi_{i}(x)=\varphi_{i}^{(m, m)}\left(x_{i_{1}}, \ldots, x_{i_{m}}\right)+\sum_{j \in I \backslash I_{m}} \varphi_{i j}\left(x_{j}\right) ;$

(2) $\forall i \in I \backslash I_{m}$ and $\forall x \in U$, one has $\varphi_{i}^{(m, m)}\left(x_{i_{1}}, \ldots, x_{i_{m}}\right)=0$ and $\varphi_{i j}\left(x_{j}\right)=0, \forall j \neq \sigma(i)$;

(3) $\forall i \in I \backslash I_{m}$, the function $g_{i}=\varphi_{i, \sigma(i)}$ is constant or injective derivable; moreover, $\forall x=\left(x_{j}: j \in I \backslash I_{m}\right) \in$ $\prod_{j \in I \backslash I_{m}} A_{j}$, there exists $\prod_{i \in \mathcal{F}_{\varphi}}(-1)^{|i|+|\sigma(i)|} g_{i}^{\prime}\left(x_{\sigma(i)}\right) \in$ $\mathbf{R}^{*}$, where $\mathscr{I}_{\varphi}=\left\{i \in I \backslash I_{m}: g_{i}\right.$ is injective derivable $\}$.

If $\sigma(i)=i, \forall i \in I \backslash I_{m}, \varphi$ is called $m$-standard; moreover, if the sequence $\left\{(-1)^{|i|+|\sigma(i)|} g_{i}^{\prime}\left(x_{\sigma(i)}\right)\right\}_{i \in \mathcal{Y}_{\varphi}}$ converges uniformly to 1 , with respect to $x=\left(x_{j}: j \in I \backslash I_{m}^{\varphi}\right) \in \prod_{j \in I \backslash I_{m}} A_{j}$, then $\varphi$ is called strongly $(m, \sigma)$-standard.

Furthermore, $\forall n \geq m$, define the function $\varphi^{(n, n)}$ : $\pi_{I, I_{n}}(U) \rightarrow \mathbf{R}^{n}$ by $\varphi^{(n, n)}(\mathbf{x})=\left(\varphi_{i_{1}}^{(n, n)}(\mathbf{x}), \ldots, \varphi_{i_{n}}^{(n, n)}(\mathbf{x})\right)$, where

$$
\begin{aligned}
\varphi_{i}^{(n, n)}(\mathbf{x})=\varphi_{i}^{(m, m)}\left(x_{i_{1}}, \ldots, x_{i_{m}}\right)+\sum_{j \in I_{n} \backslash I_{m}} \varphi_{i j}\left(x_{j}\right), \\
\forall \mathbf{x}=\left(x_{j}: j \in I_{n}\right) \in \pi_{I, I_{n}}(U), \forall i \in I_{n} .
\end{aligned}
$$

Finally, define the $(m, \sigma)$-standard function $\varphi^{(n)}: U \subset E_{I} \rightarrow$ $E_{I}$ in the following manner:

$$
\begin{array}{r}
\varphi_{i}^{(n)}(x)=\varphi_{i}^{(n, n)}\left(x_{i_{1}}, \ldots, x_{i_{n}}\right), \\
\forall x=\left(x_{j}: j \in I\right) \in U, \quad \forall i \in I_{m}, \\
\varphi_{i}^{(n)}(x)=\varphi_{i}(x), \\
\forall x=\left(x_{j}: j \in I\right) \in U, \forall i \in I \backslash I_{m},
\end{array}
$$

and indicate $\varphi^{(m)}$ by $\bar{\varphi}$.

Remark 29. Let $\varphi: U \subset E_{I} \rightarrow E_{I}$ be a $(m, \sigma)$-standard function. Then,

(1) if $\sigma$ is injective, for any $n \in \mathbf{N}, n \geq m, \varphi$ is $(n, \sigma)$ standard;

(2) $\sigma$ is biunique if and only if $\sigma(i)=i, \forall i \in I \backslash I_{m}$;

(3) if $\prod_{j \in I \backslash I_{m}} A_{j} \subset E_{I \backslash I_{m}}$, there exist $a \in \mathbf{R}^{+}$and $m_{0} \in \mathbf{N}$, $m_{0} \geq m$, such that, for any $j \in I \backslash I_{m_{0}}$, one has $A_{j} \subset$ $(-a, a)$. 
Proof. Points (1) and (2) follow from the fact that $\sigma$ is increasing. Moreover, the proof of the point (3) is trivial.

Remark 30. Let $\varphi: U \subset E_{I} \rightarrow E_{I}$ be a $(m, \sigma)$-standard function such that $\prod_{j \in I \backslash I_{m}} A_{j} \subset E_{I \backslash I_{m}}$, and $\sigma$ is injective; then, there exists $m_{1} \in \mathbf{N}, m_{1} \geq m$, such that, for any $i \in I \backslash I_{m_{1}}, g_{i}$ is bounded. In particular, if $|I|=+\infty, \varphi$ is not surjective.

Proof. Since $\prod_{j \in I \backslash I_{m}} A_{j} \subset E_{I \backslash I_{m}}$, from Remark 29, there exists $m_{0} \in \mathbf{N}, m_{0} \geq m$, such that, $\forall j \in I \backslash I_{m_{0}}$, the set $A_{j}$ is bounded; then, let $\mathscr{H}=\left\{i \in I \backslash I_{m_{0}}: g_{i}\right.$ is not bounded $\} \subset \mathscr{I}_{\varphi}$; since $\sigma$ is injective and increasing, we have $\sigma(\mathscr{H}) \subset I \backslash I_{m_{0}}$. Moreover, we have $|\mathscr{H}|<+\infty$; indeed, $\forall i \in \mathscr{H}$, the set $A_{\sigma(i)}$ is bounded, and so there exists $t_{i} \in A_{\sigma(i)}$ such that $\left|g_{i}^{\prime}\left(t_{i}\right)\right|>2$; by supposing by contradiction $|\mathscr{H}|=+\infty, \forall x=\left(x_{j}: j \in\right.$ $\left.I \backslash I_{m}\right) \in \prod_{j \in I \backslash I_{m}} A_{j}$ such that $x_{\sigma(i)}=t_{i}, \forall i \in \mathscr{H}$, we would obtain $\prod_{i \in \mathscr{I}_{\varphi}}\left|g_{i}^{\prime}\left(x_{\sigma(i)}\right)\right|=\prod_{i \in \mathscr{I}_{\varphi} \mid \mathscr{H}}\left|g_{i}^{\prime}\left(x_{\sigma(i)}\right)\right| \prod_{i \in \mathscr{H}}\left|g_{i}^{\prime}\left(t_{i}\right)\right|=$ $+\infty$ (a contradiction). Then, there exists $m_{1} \in \mathbf{N}, m_{1} \geq m$, such that, $\forall i \in I \backslash I_{m_{1}}, g_{i}$ is bounded. In particular, $\forall i \in I \backslash I_{m_{1}}$, the function $g_{i}$ is not surjective; then, if $|I|=+\infty, \varphi$ is not surjective.

Proposition 31. Let $\varphi: U \subset E_{I} \rightarrow E_{I}$ be $a(m, \sigma)$-standard function; then,

(1) suppose that $\varphi$ is injective, $\pi_{I, H}(\varphi(U)) \in \tau^{(H)}$, for any $H \subset I \backslash I_{m}$ such that $0<|H| \leq 2$, the function $\varphi_{i}$ : $U \rightarrow \mathbf{R}$ is $C^{1}$, for any $i \in I_{m}$, and $\operatorname{det} J_{\varphi^{(m, m)}}(\mathbf{x}) \neq 0$, $\forall \mathbf{x} \in U^{(m)}$; then, the functions $g_{i}$, for any $i \in I \backslash I_{m}$, and $\varphi^{(m, m)}$ are injective, and $\sigma$ is biunique.

(2) if $\varphi$ is biunique, then the functions $g_{i}$, for any $i \in I \backslash I_{m}$, $\varphi^{(m, m)}$, and $\sigma$ are biunique.

Proof of (1). Suppose that $\varphi$ is injective, $\pi_{I, H}(\varphi(U)) \in \tau^{(H)}$, for any $H \subset I \backslash I_{m}$ such that $0<|H| \leq 2$, and let $i \in I \backslash I_{m}$; we have $i \in \mathscr{I}_{\varphi}$, since otherwise we would obtain $g_{i}(x)=c$, $\forall x \in A_{\sigma(i)}$, for some $c \in \mathbf{R}$, from which $\pi_{\{i\}}(\varphi(U))=\{c\} \notin \tau=$ $\tau^{(\{i\})}$, and this should contradict the assumption; then, $g_{i}$ is injective. Moreover, $\sigma$ must be injective; in fact, by supposing by contradiction that $\sigma\left(i_{1}\right)=\sigma\left(i_{2}\right)$, for some $m<i_{1}<i_{2}$, then

$$
\begin{aligned}
& \pi_{I,\left\{i_{1}, i_{2}\right\}}(\varphi(U))=\left\{\left(y_{1}, y_{2}\right) \in \mathbf{R}^{2}: y_{1}=g_{i_{1}}(x), y_{2}\right. \\
& \left.=g_{i_{2}}(x), \text { for some } x \in A_{\sigma\left(i_{1}\right)}\right\}=\left\{\left(y_{1}, y_{2}\right)\right. \\
& \left.\in \mathbf{R}^{2}: y_{1} \in g_{i_{1}}\left(A_{\sigma\left(i_{1}\right)}\right), y_{2}=g_{i_{2}}\left(g_{i_{1}}^{-1}\left(y_{1}\right)\right)\right\} \\
& \notin \tau^{(2)}=\tau^{\left(\left\{i_{1}, i_{2}\right\}\right)}
\end{aligned}
$$

(a contradiction). Moreover, $\sigma$ is surjective; in fact, suppose by contradiction that there exists $n \in\left(I \backslash I_{m}\right) \backslash \sigma\left(I \backslash I_{m}\right)$; since $\varphi$ is injective, $\forall y \in \varphi(U)$, there is a unique $x=\left(x_{j}: j \in I\right) \in U$ such that $\varphi(x)=y$, and so

$$
\begin{aligned}
y_{i} & =\varphi_{i}(x)=\varphi_{i}^{(m, m)}\left(x_{i_{1}}, \ldots, x_{i_{m}}\right)+\varphi_{i n}\left(x_{n}\right) \\
& +\sum_{j \in I \backslash\left(I_{m} \cup\{n\}\right)} \varphi_{i j}\left(x_{j}\right), \quad \forall i \in I_{m} \Longrightarrow
\end{aligned}
$$

$$
\begin{aligned}
& \varphi_{i}^{(m, m)}\left(x_{i_{1}}, \ldots, x_{i_{m}}\right)+\varphi_{i n}\left(x_{n}\right)-y_{i} \\
& +\sum_{j \in I \backslash\left(I_{m} \cup\{n\}\right)} \varphi_{i j}\left(x_{j}\right)=0, \quad \forall i \in I_{m} .
\end{aligned}
$$

Then, consider the function $F: U^{(m)} \times A_{n} \rightarrow \mathbf{R}^{m}$ defined by

$$
\begin{aligned}
F_{i}(\mathbf{z}, t)=\varphi_{i}^{(m, m)}(\mathbf{z})+\varphi_{i n}(t)-y_{i} & \\
+ & +\sum_{j \in I \backslash\left(I_{m} \cup\{n\}\right)} \varphi_{i j}\left(x_{j}\right), \\
& \forall \mathbf{z} \in U^{(m)}, \forall t \in A_{n}, \forall i \in I_{m} .
\end{aligned}
$$

From (28) and by assumption, we have

$$
\begin{array}{r}
F\left(x_{i_{1}}, \ldots, x_{i_{m}}, x_{n}\right)=\mathbf{0}, \\
\operatorname{det} \frac{\partial F}{\partial \mathbf{z}}\left(x_{i_{1}}, \ldots, x_{i_{m}}, x_{n}\right) \neq 0 ;
\end{array}
$$

then, there exist a neighbourhood $V_{1} \subset A_{n}$ of $x_{n}$ and a neighbourhood $V_{2} \subset U^{(m)}$ of $\left(x_{i_{1}}, \ldots, x_{i_{m}}\right)$ such that, $\forall t \in V_{1}$, there exists a unique $\mathbf{z}=f(t) \stackrel{\in}{\in} V_{2}$ such that $F(\mathbf{z}, t)=\mathbf{0}$ (a contradiction with the uniqueness of $x=\left(x_{j}: j \in I\right) \in U$ such that $\varphi(x)=y)$. Finally, let $\mathbf{x}, \mathbf{y} \in U^{(m)}$ be such that $\varphi^{(m, m)}(\mathbf{x})=\varphi^{(m, m)}(\mathbf{y})$, and let $\bar{x}, \bar{y} \in U$ be such that $\bar{x}_{i}=x_{i}$ and $\bar{y}_{i}=y_{i}, \forall i \in I_{m}, \bar{x}_{i}=\bar{y}_{i}, \forall i \in I \backslash I_{m}$. We have

$$
\begin{aligned}
\varphi_{i}(\bar{x}) & =\varphi_{i}^{(m, m)}(\mathbf{x})+\sum_{j \in I \backslash I_{m}} \varphi_{i j}\left(\bar{x}_{j}\right) \\
& =\varphi_{i}^{(m, m)}(\mathbf{y})+\sum_{j \in I \backslash I_{m}} \varphi_{i j}\left(\bar{y}_{j}\right)=\varphi_{i}(\bar{y}),
\end{aligned}
$$

$\forall i \in I_{m}$

$$
\varphi_{i}(\bar{x})=g_{i}\left(\bar{x}_{\sigma(i)}\right)=g_{i}\left(\bar{y}_{\sigma(i)}\right)=\varphi_{i}(\bar{y}), \quad \forall i \in I \backslash I_{m},
$$

from which $\varphi(\bar{x})=\varphi(\bar{y})$; then, since $\varphi$ is injective, we have $\bar{x}=\bar{y}$, and so $\mathbf{x}=\mathbf{y}$. Then, the function $\varphi^{(m, m)}$ is injective.

Proof of (2). Suppose that $g_{i}$ is injective, $\forall i \in I \backslash I_{m}$, and let $x, y \in U$ be such that $\varphi(x)=\varphi(y)$; then, $\forall i \in I \backslash I_{m}$ we have $g_{i}\left(x_{\sigma(i)}\right)=\varphi_{i}(x)=\varphi_{i}(y)=g_{i}\left(y_{\sigma(i)}\right)$, from which $x_{\sigma(i)}=y_{\sigma(i)}$; then, if $\sigma$ is biunique, from Remark 29, we have $\sigma(i)=i$, and so $\left(x_{i}: i \in I \backslash I_{m}\right)=\left(y_{i}: i \in I \backslash I_{m}\right)$. This implies that

$$
\begin{aligned}
\varphi_{i}^{(m, m)}\left(x_{i_{1}}, \ldots, x_{i_{m}}\right) & =\varphi_{i}(x)-\sum_{j \in I \backslash I_{m}} \varphi_{i j}\left(x_{j}\right) \\
& =\varphi_{i}(y)-\sum_{j \in I \backslash I_{m}} \varphi_{i j}\left(y_{j}\right) \\
& =\varphi_{i}^{(m, m)}\left(y_{i_{1}}, \ldots, y_{i_{m}}\right), \\
\varphi^{(m, m)}\left(x_{i_{1}}, \ldots, x_{i_{m}}\right) & =\varphi^{(m, m)}\left(y_{i_{1}}, \ldots, y_{i_{m}}\right),
\end{aligned}
$$


and so, if $\varphi^{(m, m)}$ is injective, we have $\left(x_{i_{1}}, \ldots, x_{i_{m}}\right)=$ $\left(y_{i_{1}}, \ldots, y_{i_{m}}\right)$; then, $x=y$; that is, $\varphi$ is injective.

Proposition 32. Let $\varphi: U \subset E_{I} \rightarrow E_{I}$ be a $(m, \sigma)$-standard function; then:

(1) Suppose that $\varphi$ is biunique, the function $\varphi_{i}: U \rightarrow \mathbf{R}$ is $C^{1}$, for any $i \in I_{m}$, and $\operatorname{det} J_{\varphi^{(m, m)}}(\mathbf{x}) \neq 0$, for any $\mathbf{x} \in U^{(m)}$. Then, the functions $g_{i}$, for any $i \in I \backslash I_{m}, \sigma$ and $\varphi^{(m, m)}$ are biunique.

(2) If the functions $g_{i}$, for any $i \in I \backslash I_{m}, \sigma$ and $\varphi^{(m, m)}$ are biunique, then $\varphi$ is biunique.

Proof of (1). If $\varphi$ is biunique, $\forall H \subset I \backslash I_{m}$ such that $0<|H| \leq 2$; we have $\pi_{I, H}(\varphi(U))=\mathbf{R}^{H} \in \tau^{(H)}$; then, from Proposition 31, the functions $g_{i}, \forall i \in I \backslash I_{m}$, and $\varphi^{(m, m)}$ are injective, and $\sigma$ is biunique; thus, $\forall i \in I \backslash I_{m}$, we have $\sigma(i)=i$. Moreover, $\forall i \in I \backslash I_{m}, g_{i}$ is surjective, since $\varphi$ is surjective. Furthermore, $\forall \mathbf{y}=\left(y_{i_{1}}, \ldots, y_{i_{m}}\right) \in \mathbf{R}^{m}$, let $\left(x_{j}: j \in I \backslash I_{m}\right) \in\left(\prod_{j \in I \backslash I_{m}} A_{j}\right) \cap$ $E_{I \backslash I_{m}}$ and let $\bar{y} \in E_{I}$ be such that

$$
\begin{aligned}
& \bar{y}_{i}=y_{i}+\sum_{j \in I \backslash I_{m}} \varphi_{i j}\left(x_{j}\right), \quad \forall i \in I_{m}, \\
& \bar{y}_{i}=g_{i}\left(x_{i}\right), \quad \forall i \in I \backslash I_{m} ;
\end{aligned}
$$

moreover, let $\bar{x}=\varphi^{-1}(\bar{y}) \in U$, from which $\bar{y}_{i}=g_{i}\left(\bar{x}_{i}\right), \forall i \epsilon$ $I \backslash I_{m}$. Since $g_{i}$ is injective, we have $\bar{x}_{i}=x_{i}, \forall i \in I \backslash I_{m}$; then, $\forall i \in I_{m}$, we have

$$
\begin{aligned}
& \varphi_{i}^{(m, m)}\left(\bar{x}_{i_{1}}, \ldots, \bar{x}_{i_{m}}\right)+\sum_{j \in I \backslash I_{m}} \varphi_{i j}\left(x_{j}\right)=\bar{y}_{i} \\
& =y_{i}+\sum_{j \in I \backslash I_{m}} \varphi_{i j}\left(x_{j}\right) \Longrightarrow \\
& y_{i}=\varphi_{i}^{(m, m)}\left(\bar{x}_{i_{1}}, \ldots, \bar{x}_{i_{m}}\right) \Longrightarrow \\
& \mathbf{y}=\varphi^{(m, m)}(\mathbf{x}),
\end{aligned}
$$

where $\mathbf{x}=\left(\bar{x}_{i_{1}}, \ldots, \bar{x}_{i_{m}}\right) \in U^{(m)}$; then, $\varphi^{(m, m)}$ is surjective.

Proof of (2). If the functions $g_{i}, \forall i \in I \backslash I_{m}, \sigma$ and $\varphi^{(m, m)}$ are biunique, from Proposition 31, we obtain that $\varphi$ is injective. Moreover, $\forall y \in E_{I}$, define $x \in U^{(m)} \times \prod_{j \in I \backslash I_{m}} A_{j}$ in the following manner:

$$
\begin{aligned}
x_{i} & =g_{i}^{-1}\left(y_{i}\right) \in A_{i}, \quad \forall i \in I \backslash I_{m}, \\
\left(x_{i_{1}}, \ldots, x_{i_{m}}\right) & =\left(\varphi^{(m, m)}\right)^{-1}\left(z_{i_{1}}, \ldots, z_{i_{m}}\right) \in U^{(m)},
\end{aligned}
$$

where

$$
z_{i}=y_{i}-\sum_{j \in I \backslash I_{m}} \varphi_{i j}\left(x_{j}\right), \quad \forall i \in I_{m} .
$$

Let $x_{0}=\left(x_{0, i}: i \in I\right) \in U ; \forall i \in I \backslash I_{m}$, we have

$$
\begin{aligned}
\left|x_{i}\right| & =\left|g_{i}^{-1}\left(y_{i}\right)-x_{0, i}+x_{0, i}\right| \\
& \leq\left|g_{i}^{-1}\left(y_{i}\right)-g_{i}^{-1}\left(g_{i}\left(x_{0, i}\right)\right)\right|+\left|x_{0, i}\right| ;
\end{aligned}
$$

moreover, the function $g_{i}^{-1}: \mathbf{R} \rightarrow A_{i}$ is derivable, and

$$
\left(g_{i}^{-1}\right)^{\prime}(t)=\frac{1}{g_{i}^{\prime}\left(g_{i}^{-1}(t)\right)} \in \mathbf{R}^{*}, \quad \forall i \in I \backslash I_{m}, \forall t \in \mathbf{R} ;
$$

then, the Lagrange theorem implies that, for some $\xi_{i} \epsilon$ $\left(\min \left\{y_{i}, g_{i}\left(x_{0, i}\right)\right\}, \max \left\{y_{i}, g_{i}\left(x_{0, i}\right)\right\}\right)$, we have

$$
\begin{aligned}
& \left|g_{i}^{-1}\left(y_{i}\right)-g_{i}^{-1}\left(g_{i}\left(x_{0, i}\right)\right)\right| \\
& \quad=\left|\left(g_{i}^{-1}\right)^{\prime}\left(\xi_{i}\right)\right|\left|y_{i}-g_{i}\left(x_{0, i}\right)\right| ;
\end{aligned}
$$

thus, from (37) and (38), we obtain

$$
\left|x_{i}\right| \leq \frac{\left|y_{i}-g_{i}\left(x_{0, i}\right)\right|}{\left|g_{i}^{\prime}\left(g_{i}^{-1}\left(\xi_{i}\right)\right)\right|}+\left|x_{0, i}\right| \text {. }
$$

Moreover, we have $\prod_{i \in I \backslash I_{m}}\left|g_{i}^{\prime}\left(g_{i}^{-1}\left(\xi_{i}\right)\right)\right|=$ $\prod_{i \in \mathcal{I}_{\varphi}}\left|g_{i}^{\prime}\left(g_{i}^{-1}\left(\xi_{i}\right)\right)\right| \in \mathbf{R}^{+}$, from which there exists $i_{0} \in I \backslash I_{m}$ such that $\forall i \in I \backslash I_{m}, i>i_{0}$, we have $\left|g_{i}^{\prime}\left(g_{i}^{-1}\left(\xi_{i}\right)\right)\right|>1 / 2$; then, there exists $c \in \mathbf{R}^{+}$such that $\sup _{i \in I \backslash I_{m}}\left|g_{i}^{\prime}\left(g_{i}^{-1}\left(\xi_{i}\right)\right)\right|^{-1} \leq c$, and so formula (40) implies

$$
\sup _{i \in I \backslash I_{m}}\left|x_{i}\right| \leq c\left(\|y\|_{I}+\left\|\varphi\left(x_{0}\right)\right\|_{I}\right)+\left\|x_{0}\right\|_{I}<+\infty ;
$$

then, we have $x \in E_{I}$, from which $x \in U$. Finally, it is easy to prove that $\varphi(x)=y$, and so $\varphi$ is surjective.

Remark 33. Let $\varphi: U \subset E_{I} \rightarrow E_{I}$ be a $(m, \sigma)$-standard function such that $\varphi_{i j}\left(x_{j}\right)=0$, for any $x_{j} \in A_{j}$, for any $i \in I_{m}$, and for any $j \in I \backslash I_{m}$; then,

(1) if $\varphi$ is injective, and $\pi_{I, H}(\varphi(U)) \in \tau^{(H)}$, for any $H \subset$ $I \backslash I_{m}$ such that $0<|H| \leq 2$, then the functions $g_{i}$, for any $i \in I \backslash I_{m}$, and $\varphi^{(m, m)}$ are injective, and $\sigma$ is biunique.

(2) if $\varphi$ is biunique, then the functions $g_{i}$, for any $i \in I \backslash I_{m}$, $\varphi^{(m, m)}$ and $\sigma$ are biunique.

Proof of (1). Suppose that $\varphi$ is injective; by proceeding as in the proof of Proposition 31, we have that the functions $g_{i}, \forall i \in$ $I \backslash I_{m}, \varphi^{(m, m)}$, and $\sigma$ are injective. Moreover, $\sigma$ is surjective; in fact, suppose by contradiction that there exists $n \in\left(I \backslash I_{m}\right) \backslash$ $\sigma\left(I \backslash I_{m}\right)$, and let $x=\left(x_{j}: j \in I\right)$; moreover, $\forall t \in A_{n}, t \neq x_{n}$, let $\bar{x}=\left(\bar{x}_{j}: j \in I\right) \in U$ be the sequence defined by $\bar{x}_{j}=x_{j}$, $\forall j \neq n$, and $\bar{x}_{n}=t$; we have

$$
\begin{aligned}
\varphi_{i}(x) & =\varphi_{i}^{(m, m)}\left(x_{i_{1}}, \ldots, x_{i_{m}}\right)=\varphi_{i}^{(m, m)}\left(\bar{x}_{i_{1}}, \ldots, \bar{x}_{i_{m}}\right) \\
& =\varphi_{i}(\bar{x}), \quad \forall i \in I_{m}, \\
\varphi_{i}(x) & =g_{i}\left(x_{\sigma(i)}\right)=g_{i}\left(\bar{x}_{\sigma(i)}\right)=\varphi_{i}(\bar{x}), \quad \forall i \in I \backslash I_{m} ;
\end{aligned}
$$

thus, we have $\varphi(x)=\varphi(\bar{x})$, and so $\varphi$ is not injective (a contradiction).

Proof of (2). Since $\varphi$ is surjective, the functions $g_{i}, \forall i \in I \backslash I_{m}$, and $\varphi^{(m, m)}$ are surjective; moreover, $\forall H \subset I \backslash I_{m}$ such that $0<|H| \leq 2$, we have $\pi_{I, H}(\varphi(U))=\mathbf{R}^{H} \in \tau^{(H)}$; then, since $\varphi$ is injective, from point 1 , the functions $g_{i}, \forall i \in I \backslash I_{m}$, and $\varphi^{(m, m)}$ are injective, and $\sigma$ is biunique. 
Corollary 34. Let $\varphi: U \subset E_{I} \rightarrow E_{I}$ be a $(m, \sigma)$-standard function such that $\pi_{I, H}(\varphi(U)) \in \tau^{(H)}$, for any $H \subset I \backslash I_{m}$ such that $0<|H| \leq 2$, and $\bar{\varphi}$ is injective; then, the functions $\varphi, \varphi^{(n)}$, and $\varphi^{(n, n)}$, for any $n \in \mathbf{N}, n \geq m$, are injective.

Proof. Observe that $\bar{\varphi}$ is $(m, \sigma)$-standard, and $\pi_{I, H}(\bar{\varphi}(U))=$ $\pi_{I, H}(\varphi(U)) \in \tau^{(H)}, \forall H \subset I \backslash I_{m}$ such that $0<|H| \leq$ 2; then, from Remark 33, we have that the functions $g_{i}$, $\forall i \in I \backslash I_{m}$, and $\varphi^{(m, m)}$ are injective, and $\sigma$ is biunique; then, from Proposition 31, $\varphi$ is injective; analogously, since $\forall n \in \mathbf{N}, n \geq m$, the function $\varphi^{(n)}$ is $(n, \sigma)$-standard, from Proposition $31 \varphi^{(n)}$ is injective, Moreover, we have $\pi_{I, H}\left(\varphi^{(n)}(U)\right)=\pi_{I, H}(\varphi(U)) \in \tau^{(H)}, \forall H \subset I \backslash I_{n}$ such that $0<|H| \leq 2$; then, from Remark 33, $\varphi^{(n, n)}=\left(\varphi^{(n)}\right)^{(n, n)}$ is injective.

Proposition 35. Let $\varphi: U \subset E_{I} \rightarrow E_{I}$ be a function $C^{1}$ in $x_{0}=\left(x_{0, j}: j \in I\right) \in U$ and $(m, \sigma)$-standard. Then, for any $n \geq m$, the function $\varphi^{(n, n)}: \pi_{I, I_{n}}(U) \rightarrow \mathbf{R}^{n}$ is $C^{1}$ in $\left(x_{0, j}:\right.$ $\left.j \in I_{n}\right)$, the function $\varphi^{(n)}: U \subset E_{I} \rightarrow E_{I}$ is $C^{1}$ in $x_{0}$, there exists the function $J_{\varphi^{(n)}}\left(x_{0}\right): E_{I} \rightarrow E_{I}$, and it is continuous. Moreover, if $\varphi$ is $C^{1}$ in $x_{0}$ and strongly $(m, \sigma)$-standard, then $\varphi^{(n)}$ is differentiable in $x_{0}$. Finally, if $\varphi$ is strongly $C^{1}$ in $x_{0}$ and strongly $(m, \sigma)$-standard, then $\varphi$ is differentiable in $x_{0}$.

Proof. By assumption, there exists a neighbourhood $V=$ $\prod_{j \in I} V_{j} \in \tau_{\|\cdot\|_{I}}(U)$ of $x_{0}$ such that, $\forall i, j \in I$, there exists the function $x \rightarrow \partial \varphi_{i}(x) / \partial x_{j}$ on $V$, and this function is continuous in $x_{0}$; then, $\forall \mathbf{x}=\left(x_{j}: j \in I_{n}\right) \in \prod_{j \in I_{n}} V_{j}$, let $x \in V$ such that $\left(x_{j}: j \in I_{n}\right)=\mathbf{x}$; since $\varphi$ is a $(m, \sigma)$-standard function, $\forall i, j \in I_{n}$, we have

$$
\frac{\partial \varphi_{i}^{(n, n)}(\mathbf{x})}{\partial x_{j}}=\frac{\partial \varphi_{i}(x)}{\partial x_{j}}
$$

from which $\varphi^{(n, n)}$ is $C^{1}$ in $\left(x_{0, j}: j \in I_{n}\right)$. Moreover, $\forall x \in V$, we have

$$
\begin{aligned}
& \frac{\partial \varphi_{i}^{(n)}(x)}{\partial x_{j}} \\
& = \begin{cases}\frac{\partial \varphi_{i}(x)}{\partial x_{j}} & \text { if }(i, j) \in\left(I \times I_{m}\right) \cup\left(\left(I \backslash I_{m}\right) \times\left(I \backslash I_{m}\right)\right) \\
0 & \text { if }(i, j) \in I_{m} \times\left(I \backslash I_{m}\right),\end{cases}
\end{aligned}
$$

and so $\varphi^{(n)}$ is $C^{1}$ in $x_{0}$. Furthermore, $\forall i \in I$, the function $\varphi_{i}^{(n)}: U \subset E_{I} \rightarrow \mathbf{R}$ is differentiable in $x_{0}$, since $\varphi_{i}^{(n)}$ depends only on a finite number of variables, and so there exists the function $J_{\varphi_{i}^{(n)}}\left(x_{0}\right): E_{I} \rightarrow \mathbf{R}$; moreover, since $\forall i \in I \backslash I_{m}$ we have $\left\|J_{\varphi_{i}^{(n)}}\left(x_{0}\right)\right\|=\left|g_{i}^{\prime}\left(x_{0, \sigma(i)}\right)\right|$ and since the sequence $\left\{\left|g_{i}^{\prime}\left(x_{0, \sigma(i)}\right)\right|\right\}_{i \in \mathscr{I}_{\varphi}}$ is convergent, this implies that $\sup _{i \in I \backslash I_{m}}\left|g_{i}^{\prime}\left(x_{0, \sigma(i)}\right)\right|<+\infty$, and so $\sup _{i \in I}\left\|J_{\varphi_{i}^{(n)}}\left(x_{0}\right)\right\|<+\infty$; then, there exists the function $J_{\varphi^{(n)}}\left(x_{0}\right): E_{I} \rightarrow E_{I}$, and it is continuous.

Moreover, suppose that $\varphi$ is $C^{1}$ in $x_{0}$ and strongly $(m, \sigma)$-standard; then, the sequence $\left\{(-1)^{|i|+|\sigma(i)|} g_{i}^{\prime}\left(x_{\sigma(i)}\right)\right\}_{i \in \mathcal{F}_{\varphi}}$ converges uniformly to 1 , with respect to $x=\left(x_{j}: j \in\right.$ $\left.I \backslash I_{m}\right) \in \prod_{j \in I \backslash I_{m}} A_{j}$; thus, $\forall \varepsilon>0$, there exists $\bar{i} \in I \backslash I_{m}$ such that, $\forall i \in \mathscr{I}_{\varphi}, i>\bar{i}$, and $\forall x \in U$, we have

$$
\left|(-1)^{|i|+|\sigma(i)|} g_{i}^{\prime}\left(x_{\sigma(i)}\right)-1\right|<\frac{\varepsilon}{2} \text {. }
$$

Observe that, since $\forall i \leq \bar{i}$ the function $\varphi_{i}^{(n)}$ is differentiable in $x_{0}$, there exists a neighbourhood $N \in \tau_{\|\cdot\|_{I}}(U)$ of $x_{0}$ such that, $\forall x \in N \backslash\left\{x_{0}\right\}$, we have

$$
\sup _{i \in I: i \leq \bar{i}} \frac{\left|\varphi_{i}^{(n)}(x)-\varphi_{i}^{(n)}\left(x_{0}\right)-J_{\varphi_{i}^{(n)}}\left(x_{0}\right)\left(x-x_{0}\right)\right|}{\left\|x-x_{0}\right\|_{J}}<\varepsilon .
$$

Moreover, $\forall i \in I$ such that $i>\bar{i}, g_{i}$ is derivable in $A_{\sigma(i)}$ and so, from the Lagrange theorem, there exists $\xi_{i} \in$ $\left(\min \left\{x_{0, \sigma(i)}, x_{\sigma(i)}\right\}, \max \left\{x_{0, \sigma(i)}, x_{\sigma(i)}\right\}\right)$ such that

$$
g_{i}\left(x_{\sigma(i)}\right)-g_{i}\left(x_{0, \sigma(i)}\right)=g_{i}^{\prime}\left(\xi_{i}\right)\left(x_{\sigma(i)}-x_{0, \sigma(i)}\right),
$$

from which

$$
\begin{aligned}
& \frac{\left|\varphi_{i}^{(n)}(x)-\varphi_{i}^{(n)}\left(x_{0}\right)-J_{\varphi_{i}^{(n)}}\left(x_{0}\right)\left(x-x_{0}\right)\right|}{\left\|x-x_{0}\right\|_{J}} \\
& \quad=\frac{\left|g_{i}\left(x_{\sigma(i)}\right)-g_{i}\left(x_{0, \sigma(i)}\right)-g_{i}^{\prime}\left(x_{0, \sigma(i)}\right)\left(x_{\sigma(i)}-x_{0, \sigma(i)}\right)\right|}{\left\|x-x_{0}\right\|_{J}} \\
& \quad=\frac{\left|(-1)^{|i|+|\sigma(i)|} g_{i}^{\prime}\left(\xi_{i}\right)-(-1)^{|i|+|\sigma(i)|} g_{i}^{\prime}\left(x_{0, \sigma(i)}\right)\right|\left|x_{\sigma(i)}-x_{0, \sigma(i)}\right|}{\left\|x-x_{0}\right\|_{J}} \\
& \leq\left(\left|(-1)^{|i|+|\sigma(i)|} g_{i}^{\prime}\left(\xi_{i}\right)-1\right|+\left|(-1)^{|i|+|\sigma(i)|} g_{i}^{\prime}\left(x_{0, \sigma(i)}\right)-1\right|\right) \\
& \cdot 1_{\mathcal{F}_{\varphi}}(i)<\varepsilon .
\end{aligned}
$$

Then, from (46) and (48), we have

$$
\frac{\left\|\varphi^{(n)}(x)-\varphi^{(n)}\left(x_{0}\right)-J_{\varphi^{(n)}}\left(x_{0}\right)\left(x-x_{0}\right)\right\|_{I}}{\left\|x-x_{0}\right\|_{J}}<\varepsilon,
$$

and $\operatorname{so} \varphi^{(n)}$ is differentiable in $x_{0}$.

Finally, if $\varphi$ is strongly $C^{1}$ in $x_{0}$ and strongly $(m, \sigma)$ standard, the function $\psi=\varphi-\bar{\varphi}: U \subset E_{I} \rightarrow E_{I}$ given by

$$
\psi_{i}(x)= \begin{cases}\sum_{j \in I \backslash I_{m}} \varphi_{i j}\left(x_{j}\right) & \forall i \in I_{m}, \forall x \in U \\ 0 & \forall i \in I \backslash I_{m}, \forall x \in U\end{cases}
$$

is strongly $C^{1}$ in $x_{0}$, and so it is differentiable in $x_{0}$ from Proposition 27; then, since $\bar{\varphi}$ is differentiable in $x_{0}$, this is true for $\varphi=\psi+\bar{\varphi}$ too, from Remark 14 .

Proposition 36. Let $\varphi: U \subset E_{I} \rightarrow E_{I}$ be a function $C^{1}$ and $(m, \sigma)$-standard; then, $\varphi: U \rightarrow \mathbf{R}^{I}$ is $\left(\mathscr{B}^{(I)}(U), \mathscr{B}^{(I)}\right)$ measurable.

Proof. Let $i \in I$, and let $n \in \mathbf{N}, n \geq \max \{|i|, m\}$; from Proposition 35, the function $\varphi_{i}^{(n, n)}: \pi_{I, I_{n}}(U) \rightarrow \mathbf{R}$ is $C^{1}$. 
Then, $\forall B \in \tau$, we have $\left(\varphi_{i}^{(n, n)}\right)^{-1}(B) \in \tau^{(n)} \subset \mathscr{B}^{(n)}$; moreover, if we consider $\varphi_{i}^{(n, n)}$ as a function from $U$ to $\mathbf{R}$, we have $\pi_{I, I_{n}}\left(\left(\varphi_{i}^{(n, n)}\right)^{-1}(B)\right)=\mathbf{R}^{I \backslash I_{n}}$, and so $\left(\varphi_{i}^{(n, n)}\right)^{-1}(B) \in \mathscr{B}^{(I)}$; then, since $\sigma(\tau)=\mathscr{B}, \forall B \in \mathscr{B}$, we have $\left(\varphi_{i}^{(n, n)}\right)^{-1}(B) \in \mathscr{B}^{(I)}$, and so $\left(\varphi_{i}^{(n, n)}\right)^{-1}(B) \in \mathscr{B}^{(I)}(U)$. Moreover, since $\lim _{n \rightarrow+\infty} \varphi_{i}^{(n, n)}=\varphi_{i}$, the function $\varphi_{i}$ is $\left(\mathscr{B}^{(I)}(U), \mathscr{B}\right)$-measurable. Let

$$
\Sigma(I)=\left\{B=\prod_{i \in I} B_{i}: B_{i} \in \mathscr{B}, \forall i \in I\right\} ;
$$

$\forall B \in \Sigma(I)$, we have

$$
\varphi^{-1}(B)=\bigcap_{i \in I}\left(\varphi_{i}\right)^{-1}\left(B_{i}\right) \in \mathscr{B}^{(I)}(U) .
$$

Finally, since $\sigma(\Sigma(I))=\mathscr{B}^{(I)}, \forall B \in \mathscr{B}^{(I)}$, we have $\varphi^{-1}(B) \epsilon$ $\mathscr{B}^{(I)}(U)$.

Proposition 37. Let $\varphi: U \subset E_{I} \rightarrow E_{I}$ be a $(m, \sigma)$-standard function, such that $\bar{\varphi}: U \rightarrow \bar{\varphi}(U)$ is a homeomorphism. Then, the functions $\varphi^{(m, m)}: U^{(m)} \rightarrow \varphi^{(m, m)}\left(U^{(m)}\right)$ and $g_{i}: A_{i} \rightarrow$ $g_{i}\left(A_{i}\right), \forall i \in I \backslash I_{m}$, are homeomorphisms, and $\sigma$ is biunique.

Proof. Since $\bar{\varphi}$ is a homeomorphism, we have $\bar{\varphi}(U) \in \tau_{\|\cdot\|_{I}}$; then, from Proposition 6, $\forall H \subset I \backslash I_{m}$ such that $0<$ $|H| \leq 2$, we have $\pi_{I, H}(\bar{\varphi}(U)) \in \tau_{\|\cdot\|_{H}}=\tau^{(H)}$; thus, since $\bar{\varphi}$ is injective, from Remark 33, the functions $g_{i}, \forall i \in$ $I \backslash I_{m}$, and $\varphi^{(m, m)}$ are injective, and $\sigma$ is biunique; then, the functions $g_{i}$ and $g_{i}^{-1}, \forall i \in I \backslash I_{m}$, are derivable, and so they are continuous. Moreover, we have $\varphi^{(m, m)}\left(U^{(m)}\right)=$ $\pi_{I, I_{m}}(\bar{\varphi}(U)) \in \tau^{(m)}, g_{i}\left(A_{i}\right)=\pi_{I,\{i\}}(\bar{\varphi}(U)) \in \tau, \forall i \in$ $I \bigwedge^{\prime} I_{m}$. Finally, from Remark 10, the function $\left(\pi_{I, I_{m}} \circ \varphi\right)$ : $\left(U, \tau_{\|\cdot\|_{I}}(U)\right) \rightarrow\left(\mathbf{R}^{m}, \tau^{(m)}\right)$ is continuous; then, $\forall B \in \tau^{(m)}$, we have $\left(\varphi^{(m, m)}\right)^{-1}(B) \times \prod_{j \in I \backslash I_{m}} A_{j}=\left(\pi_{I, I_{m}} \circ \varphi\right)^{-1}(B) \epsilon$ $\tau_{\|\cdot\|_{I}}(U)$, from which $\left(\varphi^{(m, m)}\right)^{-1}(B) \in \tau^{(m)}\left(U^{(m)}\right)$; then, $\varphi^{(m, m)}$ is continuous; analogously, we can prove that the function $\left(\varphi^{(m, m)}\right)^{-1}$ is continuous.

Proposition 38. Let $\varphi: U \subset E_{I} \rightarrow E_{I}$ be a $(m, \sigma)$-standard function such that $\pi_{I, H}(\varphi(U)) \in \tau^{(H)}$, for any $H \subset I \backslash I_{m}$ such that $0<|H| \leq 2$. Then, $\bar{\varphi}: U \rightarrow \bar{\varphi}(U)$ is a diffeomorphism if and only if the functions $\varphi^{(m, m)}: U^{(m)} \rightarrow \varphi^{(m, m)}\left(U^{(m)}\right)$ and $g_{i}: A_{i} \rightarrow g_{i}\left(A_{i}\right), \forall i \in I \backslash I_{m}$, are diffeomorphisms, and $\sigma$ is biunique.

Proof. We have $\pi_{I, H}(\bar{\varphi}(U))=\pi_{I, H}(\varphi(U)) \in \tau^{(H)}$, for any $H \subset I \backslash I_{m}$ such that $0<|H| \leq 2$. If $\bar{\varphi}$ is a diffeomorphism, then $\bar{\varphi}$ is injective, and so, from Remark 33, the functions $g_{i}, \forall i \in I \backslash I_{m}$, and $\varphi^{(m, m)}$ are injective, and $\sigma$ is biunique. Moreover, $\bar{\varphi}$ is $C^{1}$ in $U$, and so, from Proposition $35, \varphi^{(m, m)}=$ $\bar{\varphi}^{(m, m)}$ is $C^{1}$ in $U^{(m)}=\pi_{I, I_{m}}(U)$; analogously, since $(\bar{\varphi})^{-1}$ : $\bar{\varphi}(U) \rightarrow U$ is $C^{1}$ in $\bar{\varphi}(U)$, then $\left(\varphi^{(m, m)}\right)^{-1}=\left((\bar{\varphi})^{-1}\right)^{(m, m)}$ is $C^{1}$ in $\varphi^{(m, m)}\left(U^{(m)}\right)=\pi_{I, I_{m}}(\bar{\varphi}(U)) \in \tau^{(m)}$; then, $\varphi^{(m, m)}$ is a diffeomorphism. Moreover, $\forall i \in I \backslash I_{m}, \forall x \in A_{i}$, let $\bar{x}=\left(\bar{x}_{j}: j \in I\right) \in U$ such that $\bar{x}_{i}=x$; we have $g_{i}^{\prime}(x)=\left(\partial \bar{\varphi}_{i} / \partial x_{i}\right)(\bar{x})$, and so $g_{i}$ is $C^{1}$ in $A_{i}$; analogously, $\forall i \in I \backslash I_{m}, \forall y \in g_{i}\left(A_{i}\right)$, let $\bar{y}=\left(\bar{y}_{j}: j \in I\right) \in \bar{\varphi}(U)$ such that $\bar{y}_{i}=y$; we have $\left(g_{i}^{-1}\right)^{\prime}(y)=\left(\partial\left(\bar{\varphi}^{-1}\right)_{i} / \partial y_{i}\right)(\bar{y})$, and so $g_{i}^{-1}$ is $C^{1}$ in $g_{i}\left(A_{i}\right)=\pi_{I,\{i\}}(\bar{\varphi}(U)) \in \tau$; then, $g_{i}$ is a diffeomorphism.

Conversely, if the functions $\varphi^{(m, m)}$ and $g_{i}, \forall i \in I \backslash I_{m}$, are diffeomorphisms and $\sigma$ is biunique, then $\bar{\varphi}$ is injective from Remark 33; moreover, $\forall x=\left(x_{j}: j \in I\right) \in U$, set $\mathbf{x}=\left(x_{j}: j \in\right.$ $\left.I_{m}\right) \in U^{(m)}$; we have

$$
\frac{\partial \bar{\varphi}_{i}(x)}{\partial x_{j}}= \begin{cases}\frac{\partial \varphi_{i}^{(m, m)}(\mathbf{x})}{\partial x_{j}} & \text { if }(i, j) \in\left(I_{m} \times I_{m}\right) \\ g_{i}^{\prime}\left(x_{i}\right) & \text { if } i \in I \backslash I_{m}, j=i \\ 0 & \text { otherwise, }\end{cases}
$$

and so $\bar{\varphi}$ is $C^{1}$ in $U$; analogously, $\forall y=\left(y_{j}: j \in I\right) \in \bar{\varphi}(U)$, set $\mathbf{y}=\left(y_{j}: j \in I_{m}\right) \in \pi_{I, I_{m}}(\bar{\varphi}(U))$; we have

$$
\begin{aligned}
& \frac{\partial\left(\bar{\varphi}^{-1}\right)_{i}(y)}{\partial y_{j}} \\
& \quad= \begin{cases}\frac{\partial\left(\varphi^{-1}\right)_{i}^{(m, m)}(\mathbf{y})}{\partial y_{j}} & \text { if }(i, j) \in\left(I_{m} \times I_{m}\right) \\
\left(g_{i}^{-1}\right)^{\prime}\left(y_{i}\right) & \text { if } i \in I \backslash I_{m}, j=i \\
0 & \text { otherwise, }\end{cases}
\end{aligned}
$$

and so $\bar{\varphi}^{-1}$ is $C^{1}$ in $\bar{\varphi}(U)$; then, $\bar{\varphi}$ is a diffeomorphism.

Proposition 39. Let $\varphi: U \subset E_{I} \rightarrow E_{I}$ be a $(m, \sigma)$-standard function such that $\varphi_{i}: U \rightarrow \mathbf{R}$ is $C^{1}$, for any $i \in I_{m}$; moreover, suppose that $\bar{\varphi}$ is injective and $\bar{\varphi}(U) \in \tau_{\|\cdot\|_{I}}$; then,

(1) if $\varphi^{(m, m)}:\left(U^{(m)}, \tau^{(m)}\left(U^{(m)}\right)\right) \rightarrow\left(\varphi^{(m, m)}\left(U^{(m)}\right)\right.$, $\left.\tau^{(m)}\left(\varphi^{(m, m)}\left(U^{(m)}\right)\right)\right)$ is an open function, then, for any $n \in \mathbf{N}, n \geq m$, the function $\varphi^{(n, n)}: \pi_{I, I_{n}}(U) \rightarrow$ $\varphi^{(n, n)}\left(\pi_{I, I_{n}}(U)\right)$ is a homeomorphism, and one has $\varphi^{(n)}(U) \in \tau_{\|\cdot\|_{I}}$.

(2) if $\bar{\varphi}: U \rightarrow \bar{\varphi}(U)$ is a diffeomorphism, then, for any $n \in \mathbf{N}, n \geq m$, the functions $\varphi^{(n, n)}$ : $\pi_{I, I_{n}}(U) \rightarrow \varphi^{(n, n)}\left(\pi_{I, I_{n}}(U)\right)$ and $\varphi^{(n)}: U \rightarrow \varphi^{(n)}(U)$ are diffeomorphisms.

Proof of (1). Since $\bar{\varphi}(U) \in \tau_{\|\cdot\|_{I}}, \forall H \subset I \backslash I_{m}$ such that $0<|H| \leq 2$, we have $\pi_{I, H}(\varphi(U))=\pi_{I, H}(\bar{\varphi}(U)) \in \tau_{\|\cdot\|_{H}}=\tau^{(H)}$; then, from Corollary 34, $\forall n \in \mathbf{N}, n \geq m$, the function $\varphi^{(n, n)}$ is injective. Moreover, from Remark 33, the functions $g_{i}, \forall i \in$ $I \backslash I_{m}$, and $\varphi^{(m, m)}$ are injective, and $\sigma$ is biunique. Furthermore, from Proposition 35 and the continuity of the functions $g_{i}$, $\forall i \in I_{n} \backslash I_{m}$, we have that $\varphi^{(n, n)}$ is continuous in $\pi_{I, I_{n}}(U)$. Finally, $\forall y \in \varphi^{(n, n)}\left(\pi_{I, I_{n}}(U)\right)$, let $x=\left(\varphi^{(n, n)}\right)^{-1}(y) \in \pi_{I, I_{n}}(U)$; $\forall i \in I_{n} \backslash I_{m}$, we have $x_{i}=g_{i}^{-1}\left(y_{i}\right)$; then, $\forall i \in I_{m}$,

$$
y_{i}=\varphi_{i}^{(m, m)}\left(x_{i_{1}}, \ldots, x_{i_{m}}\right)+\sum_{j \in I_{n} \backslash I_{m}} \varphi_{i j}\left(g_{j}^{-1}\left(y_{j}\right)\right),
$$


and so

$$
\begin{aligned}
\left(x_{i_{1}}, \ldots, x_{i_{m}}\right) & =\left(\varphi^{(m, m)}\right)^{-1}\left(z_{i_{1}}, \ldots, z_{i_{m}}\right), \\
x_{i} & =g_{i}^{-1}\left(y_{i}\right), \quad \forall i \in I_{n} \backslash I_{m},
\end{aligned}
$$

where

$$
z_{i}=y_{i}-\sum_{j \in I_{n} \backslash I_{m}} \varphi_{i j}\left(g_{j}^{-1}\left(y_{j}\right)\right), \quad \forall i \in I_{m} .
$$

Then, since the functions $\left(\varphi^{(m, m)}\right)^{-1}, \varphi_{i j}$, and $g_{j}^{-1}$ are continuous, the function $\left(\varphi^{(n, n)}\right)^{-1}$ is continuous too, and so $\varphi^{(n, n)}\left(\pi_{I, I_{n}}(U)\right) \in \tau^{(n)}$; then, $\varphi^{(n, n)}: \pi_{I, I_{n}}(U) \rightarrow \varphi^{(n, n)}\left(\pi_{I, I_{n}}(U)\right)$ is a homeomorphism. Furthermore, since $\bar{\varphi}(U) \in \tau_{\|\cdot\|_{I}}$, we have $\pi_{I, I \backslash I_{n}}(\bar{\varphi}(U)) \in \tau_{\|\cdot\|_{I \backslash I_{n}}}$, and so

$$
\varphi^{(n)}(U)=\varphi^{(n, n)}\left(\pi_{I, I_{n}}(U)\right) \times \pi_{I, I \backslash I_{n}}(\bar{\varphi}(U)) \in \tau_{\|\cdot\|_{I}} .
$$

Proof of (2). If $\bar{\varphi}: U \rightarrow \bar{\varphi}(U)$ is a diffeomorphism, from Proposition 38, the functions $\varphi^{(m, m)}: U^{(m)} \rightarrow \varphi^{(m, m)}\left(U^{(m)}\right)$ and $g_{i}: A_{i} \rightarrow g_{i}\left(A_{i}\right), \forall i \in I \backslash I_{m}$, are diffeomorphisms; in particular, the functions $\left(\varphi^{(m, m)}\right)^{-1}$ and $g_{i}^{-1}, \forall i \in I_{n} \backslash I_{m}$, are $C^{1}$; then, since $\forall i \in I_{m}, \forall j \in I_{n} \backslash I_{m}$, the function $\varphi_{i j}$ is $C^{1}$, from (56) and (57), we obtain that the function $\left(\varphi^{(n, n)}\right)^{-1}$ is $C^{1}$ in $\varphi^{(n, n)}\left(\pi_{I, I_{n}}(U)\right)$. Moreover, from Proposition 35 and since, $\forall i \in I_{n} \backslash I_{m}$, the functions $g_{i}$ are $C^{1}, \varphi^{(n, n)}$ is $C^{1}$ in $\pi_{I, I_{n}}(U)$, and so $\varphi^{(n, n)}$ is a diffeomorphism. Finally, since $\bar{\varphi}(U) \stackrel{\pi_{\|} I_{n}}{\epsilon} \tau_{\|\cdot\|_{I}}, \forall H \subset I \backslash I_{n}$ such that $0<|H| \leq 2$, we have $\pi_{I, H}\left(\varphi^{(n)}(U)\right)=\pi_{I, H}(\bar{\varphi}(U)) \in \tau_{\|\cdot\|_{H}}=\tau^{(H)}$; then, from Proposition $38, \varphi^{(n)}: U \rightarrow \varphi^{(n)}(U)$ is a diffeomorphism.

Remark 40. A linear function $A=\left(a_{i j}\right)_{i, j \in I}: E_{I} \rightarrow E_{I}$ is $(m, \sigma)$-standard, where $m \in \mathbf{N}^{*}$ and $\sigma: I \backslash I_{m} \rightarrow I \backslash I_{m}$ is an increasing function, if

(1) $a_{i j}=0, \forall(i, j) \notin\left(I_{m} \times I\right) \cup \bigcup_{n \in I \backslash I_{m}}\{(n, \sigma(n))\}$;

(2) there exists $\prod_{i \in I \backslash I_{m}: \lambda_{i} \neq 0} \lambda_{i} \in \mathbf{R}^{*}$, where $\lambda_{i}=$ $(-1)^{|i|+|\sigma(i)|} a_{i, \sigma(i)}, \forall i \in I \backslash I_{m}$.

Recall the following concept, defined in [1].

Definition 41. Let $A=\left(a_{i j}\right)_{i, j \in I}: E_{I} \rightarrow E_{I}$ be a linear $(m, \sigma)$ standard function; define the determinant of $A$, and call it $\operatorname{det}_{(m, \sigma)} A$, or $\operatorname{det} A$, the real number

$$
\begin{aligned}
& \operatorname{det}_{(m, \sigma)} A \\
& \quad= \begin{cases}\operatorname{det} A^{(m, m)} \prod_{i \in I \backslash I_{m}} \lambda_{i} & \text { if } \sigma \text { is biunique } \\
0 & \text { if } \sigma \text { is not biunique, }\end{cases}
\end{aligned}
$$

where $A^{(m, m)}$ is the $m \times m$ real matrix defined by $A^{(m, m)}(i, j)=$ $a_{i j}, \forall i, j \in I_{m}$.

Proposition 42. Let $A=\left(a_{i j}\right)_{i, j \in I}: E_{I} \rightarrow E_{I}$ be a linear and injective $(m, \sigma)$-standard function; then, $A$ is biunique if and only if $A$ is $\left(\tau_{\|\cdot\|_{I}}, \tau_{\|\cdot\|_{I}}\right)$-open.

Proof. From Remark 9 in [1], $A$ is continuous; then, if $A$ is biunique, from the Banach theorem of the open function, $A$ is $\left(\tau_{\|\cdot\|_{I}}, \tau_{\|\cdot\|_{I}}\right)$-open. Conversely, if $A$ is $\left(\tau_{\|\cdot\|_{I}}, \tau_{\|\cdot\|_{I}}\right)$-open, $\forall H \subset$ $I \backslash I_{m}$ such that $0<|H| \leq 2$, we have $\pi_{I, H}\left(A\left(E_{I}\right)\right) \in \tau_{\|\cdot\|_{H}}=$ $\tau^{(H)}$; moreover, since $A$ is linear, the function $A_{i}: E_{I} \rightarrow \mathbf{R}$ is $C^{1}, \forall i \in I_{m}$; furthermore, by proceeding as in the proof of Proposition 31, we obtain that $A^{(m, m)}$ is injective, and so $\operatorname{det} J_{A^{(m, m)}}(\mathbf{x}) \neq 0, \forall \mathbf{x} \in E_{I}$; then, from Proposition 31, the functions $g_{i}, \forall i \in I \backslash I_{m}$, are injective, and $\sigma$ is biunique. Thus, since the functions $A^{(m, m)}$ and $g_{i}, \forall i \in I \backslash I_{m}$, are linear, they are biunique, and so $A$ is biunique from Proposition 32 .

\section{Change of Variables' Formula}

Henceforth, we will suppose $|I|=+\infty$.

Definition 43. Let $k \in \mathbf{N}^{*}$, let $M, N \in \mathbf{R}^{+}$, let $a=\left(a_{i}: i \in I\right) \in$ $[0,+\infty)^{I}$ such that $\prod_{i \in I: a_{i} \neq 0} a_{i} \in \mathbf{R}^{+}$, and let $v=\left(v_{i}: i \in I\right) \in$ $E_{I}$; define the following sets in $\mathscr{B}_{I}$ :

$$
\begin{aligned}
E_{N, a, v}^{(k, I)} & =\mathbf{R}^{k} \times \prod_{i \in I \backslash I_{k}}\left(v_{i}-N a_{i}, v_{i}+N a_{i}\right) ; \\
E_{M, N, a, v}^{(k, I)} & =(-M, M)^{k} \times \prod_{i \in I \backslash I_{k}}\left(v_{i}-N a_{i}, v_{i}+N a_{i}\right) .
\end{aligned}
$$

Moreover, define the $\sigma$-finite measure $\lambda_{N, a, v}^{(k, I)}$ over $\left(\mathbf{R}^{I}, \mathscr{B}^{(I)}\right)$ in the following manner:

$$
\begin{aligned}
\lambda_{N, a, v}^{(k, I)} & \\
= & \left(\bigotimes_{i \in I_{k}} \frac{1}{2 N} \operatorname{Leb}\right) \\
& \otimes\left(\bigotimes_{i \in I \backslash I_{k}} \frac{1}{2 N} \operatorname{Leb}\left(\cdot \cap\left(v_{i}-N a_{i}, v_{i}+N a_{i}\right)\right)\right) .
\end{aligned}
$$

Proposition 44. Let $\varphi: U \subset E_{I} \rightarrow E_{I}$ be a $(m, \sigma)$-standard function such that $\bar{\varphi}$ is biunique; moreover, let $N \in \mathbf{R}^{+}$, let $a=\left(a_{i}: i \in I\right) \in[0,+\infty)^{I}$ such that $\prod_{i \in I: a_{i} \neq 0} a_{i} \in \mathbf{R}^{+}$, and let $v \in E_{I}$; then,

(1) there exist $b=\left(b_{i}: i \in I\right) \in[0,+\infty)^{I}$ and $z \in E_{I}$ such that $\prod_{i \in I: b_{i} \neq 0} b_{i} \in \mathbf{R}^{+}$and such that, for any $n, k \in \mathbf{N}$, $n \geq m, k \geq m$, one has

$$
\varphi^{-1}\left(E_{N, a, v}^{(k, I)}\right)=\left(\varphi^{(n)}\right)^{-1}\left(E_{N, a, v}^{(k, I)}\right)=E_{N, b, z}^{(k, I)}
$$

(2) suppose that $\varphi_{i}: U \rightarrow \mathbf{R}$ is $C^{1}$, for any $i \in I_{m}$, and the function $\varphi^{(m, m)}:\left(U^{(m)}, \tau^{(m)}\left(U^{(m)}\right)\right) \rightarrow\left(\mathbf{R}^{m}, \tau^{(m)}\right)$ 
is open; then, for any $M_{1} \in \mathbf{R}^{+}$, there exists $M_{2} \in \mathbf{R}^{+}$ such that, for any $n, k \in \mathbf{N}, n \geq m, k \geq m$, one has

$$
\begin{aligned}
& \varphi^{-1}\left(E_{M_{1}, N, a, v}^{(k, I)}\right) \subset E_{M_{2}, N, b, z}^{(k, I)}, \\
&\left(\varphi^{(n)}\right)^{-1}\left(E_{M_{1}, N, a, v}^{(k, I)}\right) \subset E_{M_{2}, N, b, z}^{(k, I)} .
\end{aligned}
$$

Proof of (1). Since $\bar{\varphi}$ is biunique, from Remark 33, the functions $g_{i}, \forall i \in I \backslash I_{m}$, and $\sigma$ are biunique. Set $b=\left(b_{i}\right.$ : $i \in I) \in[0,+\infty)^{I}, z=\left(z_{i}: i \in I\right) \in E_{I}$ such that $b_{i}=z_{i}=1$, $\forall i \in I_{m}$; moreover, define

$$
\begin{aligned}
& b_{i}=\frac{\left|g_{i}^{-1}\left(v_{i}+N a_{i}\right)-g_{i}^{-1}\left(v_{i}-N a_{i}\right)\right|}{2 N}, \quad \forall i \in I \backslash I_{m}, \\
& z_{i}=\frac{g_{i}^{-1}\left(v_{i}+N a_{i}\right)+g_{i}^{-1}\left(v_{i}-N a_{i}\right)}{2}, \quad \forall i \in I \backslash I_{m} .
\end{aligned}
$$

Observe that, $\forall i \in I \backslash I_{m}$, we have $b_{i} \neq 0$ if and only if $a_{i} \neq 0$; then, by definition of $b=\left(b_{i}: i \in I\right)$, we have

$$
\begin{aligned}
\prod_{i \in I: b_{i} \neq 0} b_{i}=\prod_{i \in I: a_{i} \neq 0} b_{i} \\
=\left(\prod_{i \in I \backslash I_{m}: a_{i} \neq 0} \frac{\left|g_{i}^{-1}\left(v_{i}+N a_{i}\right)-g_{i}^{-1}\left(v_{i}-N a_{i}\right)\right|}{2 N a_{i}}\right) \\
\\
\cdot\left(\prod_{i \in I \backslash I_{m}: a_{i} \neq 0} a_{i}\right) .
\end{aligned}
$$

Moreover, since $\forall i \in I \backslash I_{m}$ the function $g_{i}^{-1}$ is derivable on $\mathbf{R}$, if $a_{i} \neq 0$, the Lagrange theorem implies that, for some $\xi_{i} \in$ $\left(v_{i}-N a_{i}, v_{i}+N a_{i}\right)$, we have

$$
\begin{aligned}
& \frac{g_{i}^{-1}\left(v_{i}+N a_{i}\right)-g_{i}^{-1}\left(v_{i}-N a_{i}\right)}{2 N a_{i}}=\left(g_{i}^{-1}\right)^{\prime}\left(\xi_{i}\right) \\
& =\frac{1}{g_{i}^{\prime}\left(g_{i}^{-1}\left(\xi_{i}\right)\right)},
\end{aligned}
$$

from which $\prod_{i \in I: b_{i} \neq 0} b_{i} \in \mathbf{R}^{+}$. Furthermore, $\forall i \in I \backslash I_{m}$, we have

$$
\begin{aligned}
& g_{i}^{-1}\left(\left(v_{i}-N a_{i}, v_{i}+N a_{i}\right)\right) \\
& = \begin{cases}\left(g_{i}^{-1}\left(v_{i}-N a_{i}\right), g_{i}^{-1}\left(v_{i}+N a_{i}\right)\right) & \text { if } g_{i} \text { is increasing } \\
\left(g_{i}^{-1}\left(v_{i}+N a_{i}\right), g_{i}^{-1}\left(v_{i}-N a_{i}\right)\right) & \text { if } g_{i} \text { is decreasing }\end{cases} \\
& =\left(z_{i}-N b_{i}, z_{i}+N b_{i}\right),
\end{aligned}
$$

from which

$$
g_{i}\left(\left(z_{i}-N b_{i}, z_{i}+N b_{i}\right)\right)=\left(v_{i}-N a_{i}, v_{i}+N a_{i}\right) .
$$

Then, from (67) and (68), $\forall k \in \mathbf{N}, k \geq m$, we obtain

$$
\begin{aligned}
\varphi^{-1}\left(E_{N, a, v}^{(k, I)}\right) & \subset E_{N, b, z}^{(k, I)}, \\
\varphi\left(E_{N, b, z}^{(k, I)}\right) & \subset E_{N, a, v}^{(k, I)} \Longrightarrow \\
E_{N, b, z}^{(k, I)} & \subset \varphi^{-1}\left(E_{N, a, v}^{(k, I)}\right) \Longrightarrow \\
\varphi^{-1}\left(E_{N, a, v}^{(k, I)}\right) & =E_{N, b, z}^{(k, I)} .
\end{aligned}
$$

Analogously, $\forall n \in \mathbf{N}, n \geq m$, from (67) and (68), we have $\left(\varphi^{(n)}\right)^{-1}\left(E_{N, a, v}^{(k, I)}\right)=E_{N, b, z}^{(k, I)}$.

Proof of (2). Suppose that $\varphi_{i}: U \rightarrow \mathbf{R}$ is $C^{1}$, for any $i \in I_{m}$, and $\varphi^{(m, m)}:\left(U^{(m)}, \tau^{(m)}\left(U^{(m)}\right)\right) \rightarrow\left(\mathbf{R}^{m}, \tau^{(m)}\right)$ is an open function; thus, $\forall k \in \mathbf{N}, k \geq m$, and $\forall x=\left(x_{i}: i \in I\right) \in \varphi^{-1}\left(E_{M_{1}, N, a, v}^{(k, I)}\right)$, let $y=\varphi(x) \in\left(E_{M_{1}, N, a, v}^{(k, I)}\right) ; \forall i \in I \backslash I_{m}$, we have $x_{i}=g_{i}^{-1}\left(y_{i}\right)$; then, $\forall i \in I_{m}$,

$$
y_{i}=\varphi_{i}^{(m, m)}\left(x_{i_{1}}, \ldots, x_{i_{m}}\right)+\sum_{j \in I \backslash I_{m}} \varphi_{i j}\left(g_{j}^{-1}\left(y_{j}\right)\right),
$$

and so

$$
\begin{aligned}
\left(x_{i_{1}}, \ldots, x_{i_{m}}\right) & =\left(\varphi^{(m, m)}\right)^{-1}\left(z_{i_{1}}, \ldots, z_{i_{m}}\right), \\
x_{i} & =g_{i}^{-1}\left(y_{i}\right), \quad \forall i \in I \backslash I_{m}
\end{aligned}
$$

where

$$
z_{i}=y_{i}-\sum_{j \in I \backslash I_{m}} \varphi_{i j}\left(g_{j}^{-1}\left(y_{j}\right)\right), \quad \forall i \in I_{m} .
$$

Moreover, since $\forall i \in I_{m}, \forall j \in I \backslash I_{m}$, the functions $\varphi_{i j}$ and $g_{j}^{-1}$ are continuous and $\left|y_{j}\right| \leq \max \left\{M_{1},\|v\|_{I \backslash I_{m}}+N\|a\|_{I \backslash I_{m}}\right\}$, there exists $\bar{M} \in \mathbf{R}^{+}$such that

$$
\sup _{i \in I_{m}} \sum_{j \in I \backslash I_{m}}\left|\varphi_{i j}\left(g_{j}^{-1}\left(y_{j}\right)\right)\right| \leq \bar{M},
$$

and so $\left\|\left(z_{i_{1}}, \ldots, z_{i_{m}}\right)\right\|_{I_{m}} \leq M_{1}+\bar{M}$; then, since the function $\left(\varphi^{(m, m)}\right)^{-1}$ is continuous, there exists $\widetilde{M} \in \mathbf{R}^{+}$such that $\left\|\left(x_{i_{1}}, \ldots, x_{i_{m}}\right)\right\|_{I_{m}}<\widetilde{M}$; finally, if $b, z$ are the sequences defined by the point (1), by setting $M_{2}=\max \left\{\widetilde{M},\|z\|_{I \backslash I_{m}}+N\|b\|_{I \backslash I_{m}}\right\}$, from (67), we have $\varphi^{-1}\left(E_{M_{1}, N, a, v}^{(k, I)}\right) \subset E_{M_{2}, N, b, z}^{(k, I)}$. Analogously, $\forall n \in \mathbf{N}, n \geq m$, we have

$$
\sup _{i \in I_{m}} \sum_{j \in I_{n} \backslash I_{m}}\left|\varphi_{i j}\left(g_{j}^{-1}\left(y_{j}\right)\right)\right| \leq \bar{M}
$$

from which $\left(\varphi^{(n)}\right)^{-1}\left(E_{M_{1}, N, a, v}^{(k, N)}\right) \subset E_{M_{2}, N, b, z}^{(k, I)}$.

Proposition 45. Let $(S, \Sigma)$ be a measurable space, let $\mathscr{I}$ be a $\pi$-system on $S$, and let $\mu_{1}$ and $\mu_{2}$ be two measures on $(S, \Sigma), \sigma$ finite on $\mathscr{I}$; if $\sigma(\mathscr{I})=\sum$ and $\mu_{1}$ and $\mu_{2}$ coincide on $\mathscr{I}$, then $\mu_{1}$ and $\mu_{2}$ coincide on $\Sigma$.

Proof. See, for example, Theorem 10.3 in Billingsley [10].

Lemma 46. Let $k \in \mathbf{N}^{*}$, let $N \in \mathbf{R}^{+}$, let $a=\left(a_{i}: i \in I\right) \in$ $[0,+\infty)^{I}$ such that $\prod_{i \in I: a_{i} \neq 0} a_{i} \in \mathbf{R}^{+}$, and let $v=\left(v_{i}: i \in I\right) \in$ $E_{I}$; then, for any measurable function $f:\left(\mathbf{R}^{I}, \mathscr{B}^{(I)}\right) \rightarrow(\mathbf{R}, \mathscr{B})$ such that $f^{+}\left(\right.$or $\left.f^{-}\right)$is $\lambda_{N, a, v}^{(k, I)}$-integrable, one has

$$
\int_{\mathbf{R}^{I}} f d \lambda_{N, a, v}^{(k, I)}=\int_{E_{N, a, v}^{(k, I)}} f d \lambda_{N, a, v^{*}}^{(k, I)}
$$


Proof. Let $B=\prod_{i \in I} B_{i} \in \mathscr{B}^{(I)}$; by definition of $\lambda_{N, a, v}^{(k, I)}$, we have

$$
\int_{\mathbf{R}^{I}} 1_{B} d \lambda_{N, a, v}^{(k, I)}=\int_{E_{N, a, v}^{(k, I)}} 1_{B} d \lambda_{N, a, v}^{(k, I)}
$$

then, consider the measures $\mu_{1}=\lambda_{N, a, v}^{(k, I)}$ and $\mu_{2}=\lambda_{N, a, v}^{(k, I)}(\cdot \cap$ $E_{N, a, v}^{(k, I)}$ ) on $\left(\mathbf{R}^{I}, \mathscr{B}^{(I)}\right)$; from (76), $\mu_{1}$ and $\mu_{2}$ coincide on the set $\mathscr{I}=\left\{B \in \mathscr{B}^{(I)}: B=\prod_{i \in I} B_{i}\right\}$; moreover, we have $\mathbf{R}^{I}=\bigcup_{n \in \mathbf{N}}\left([-n, n]^{k} \times \mathbf{R}^{I \backslash I_{k}}\right)$, where $[-n, n]^{k} \times \mathbf{R}^{I \backslash I_{k}} \in \mathscr{I}$, $\mu_{1}\left([-n, n]^{k} \times \mathbf{R}^{I \backslash I_{k}}\right)=\mu_{2}\left([-n, n]^{k} \times \mathbf{R}^{I \backslash I_{k}}\right)<+\infty, \forall n \in \mathbf{N}$, and so $\mu_{1}$ and $\mu_{2}$ are $\sigma$-finite on $\mathscr{I}$. Then, since $\mathscr{I}$ is a $\pi$-system on $\mathbf{R}^{I}$ such that $\sigma(\mathscr{I})=\mathscr{B}^{(I)}$, from Proposition 45 , formula (76) is true $\forall B \in \mathscr{B}^{(I)}$. This implies that, if $\psi:\left(\mathbf{R}^{I}, \mathscr{B}^{(I)}\right) \rightarrow$ $([0,+\infty), \mathscr{B}([0,+\infty)))$ is a simple function, we have

$$
\int_{\mathbf{R}^{I}} \psi d \lambda_{N, a, v}^{(k, I)}=\int_{E_{N, a, v}^{(k, I)}} \psi d \lambda_{N, a, v^{*}}^{(k, I)}
$$

Then, if $f:\left(\mathbf{R}^{I}, \mathscr{B}^{(I)}\right) \rightarrow([0,+\infty), \mathscr{B}([0,+\infty)))$ is a measurable function, and $\left\{\psi_{n}\right\}_{n \in \mathbf{N}}$ is a sequence of increasing positive simple functions over $\left(\mathbf{R}^{I}, \mathscr{B}^{(I)}\right)$ such that $\lim _{n \rightarrow+\infty} \psi_{n}=f$, from Beppo Levi theorem we have

$$
\begin{aligned}
\int_{\mathbf{R}^{I}} f d \lambda_{N, a, v}^{(k, I)} & =\lim _{n \rightarrow+\infty} \int_{\mathbf{R}^{I}} \psi_{n} d \lambda_{N, a, v}^{(k, I)} \\
& =\lim _{n \rightarrow+\infty} \int_{E_{N, a, v}^{(k, I)}} \psi_{n} d \lambda_{N, a, v}^{(k, I)} \\
& =\int_{E_{N, a, v}^{(k, I)}} f d \lambda_{N, a, v}^{(k, I)}
\end{aligned}
$$

Then, for any measurable function $f:\left(\mathbf{R}^{I}, \mathscr{B}^{(I)}\right) \rightarrow(\mathbf{R}, \mathscr{B})$ such that $f^{+}$(or $f^{-}$) is $\lambda_{N, a, v}^{(k, I)}$-integrable,

$$
\begin{aligned}
\int_{\mathbf{R}^{I}} f d \lambda_{N, a, v}^{(k, I)} & =\int_{\mathbf{R}^{I}} f^{+} d \lambda_{N, a, v}^{(k, I)}-\int_{\mathbf{R}^{I}} f^{-} d \lambda_{N, a, v}^{(k, I)} \\
& =\int_{E_{N, a, v}^{(k, I)}} f^{+} d \lambda_{N, a, v}^{(k, I)}-\int_{E_{N, a, v}^{(k, I)}} f^{-} d \lambda_{N, a, v}^{(k, I)} \\
& =\int_{E_{N, a, v}^{(k, I)}} f d \lambda_{N, a, v}^{(k, I)}
\end{aligned}
$$

Now, we can prove the main result of our paper, that improves Theorem 29 in [1] and generalizes the change of variables' formula for the integration of a measurable function on $\mathbf{R}^{m}$ with values in $\mathbf{R}$ (see, e.g., the Lang's book [9]).

Theorem 47 (change of variables' formula). Let $\varphi: U \subset$ $E_{I} \rightarrow E_{I}$ be $a C^{1}$ and $(m, \sigma)$-standard function, such that the function $\bar{\varphi}: U \rightarrow E_{I}$ is a diffeomorphism; moreover, let $N \in \mathbf{R}^{+}$, let $a=\left(a_{i}: i \in I\right) \in[0,+\infty)^{I}$ such that $\prod_{i \in I: a_{i} \neq 0} a_{i} \in$ $\mathbf{R}^{+}$, let $v \in E_{I}$, and let $b \in[0,+\infty)^{I}$ and $z \in E_{I}$ defined by Proposition 44. Then, for any $k \in \mathbf{N}, k \geq m$, for any $B \in \mathscr{B}^{(I)}$, and for any measurable function $f:\left(\mathbf{R}^{I}, \mathscr{B}^{(I)}\right) \rightarrow(\mathbf{R}, \mathscr{B})$ such that $f^{+}\left(\right.$or $\left.f^{-}\right)$is $\lambda_{N, a, v}^{(k, I)}$-integrable, one has

$$
\int_{B} f d \lambda_{N, a, v}^{(k, I)}=\int_{\varphi^{-1}(B)} f(\varphi)\left|\operatorname{det} J_{\bar{\varphi}}\right| d \lambda_{N, b, z}^{(k, I)} .
$$

Proof. Let $n, k \in \mathbf{N}, n \geq k \geq m$; moreover, since $\bar{\varphi}$ is biunique, $\forall M_{1}, N \in \mathbf{R}^{+}, \forall a=\left(a_{i}: i \in I\right) \in[0,+\infty)^{I}$ such that $\prod_{i \in I: a_{i} \neq 0} a_{i} \in \mathbf{R}^{+}$, and $\forall v \in E_{I}$, let $M_{2} \in \mathbf{R}^{+}$and let $b$ and $z$ be the sequences defined by Proposition 44. Then, $\forall B=$ $\prod_{i \in I} B_{i} \in \mathscr{B}^{(I)}\left(E_{M_{1}, N, a, v}^{(k, I)}\right)$, we have $B_{i} \in \mathscr{B}\left(\left(v_{i}-N a_{i}, v_{i}+N a_{i}\right)\right)$ and $g_{i}^{-1}\left(B_{i}\right) \in \mathscr{B}\left(\left(z_{i}-N b_{i}, z_{i}+N b_{i}\right)\right), \forall i \in I \backslash I_{k}$, and so

$$
\begin{aligned}
& \int_{B} d \lambda_{N, a, v}^{(k, I)}=\int_{B_{i_{1}} \times \cdots \times B_{i_{k}} \times \prod_{q \in I \backslash I_{k}} B_{q}} d\left(\left(\bigotimes_{p=1}^{k} \frac{1}{2 N} \mathrm{Leb}\right)\right. \\
& \left.\otimes\left(\left.\bigotimes_{q \in I \backslash I_{k}} \frac{1}{2 N} \mathrm{Leb}\right|_{\mathscr{B}\left(\left(v_{q}-N a_{q}, v_{q}+N a_{q}\right)\right)}\right)\right) \\
& =\int_{B_{i_{1}} \times \cdots \times B_{i_{n}} \times \prod_{q \in I \backslash I_{n}} B_{q}} d\left(\left(\bigotimes_{p=1}^{n} \frac{1}{2 N} \mathrm{Leb}\right)\right. \\
& \left.\left.\left.\otimes \bigotimes_{q \in I \backslash I_{n}} \frac{1}{2 N} \operatorname{Leb}\right|_{\mathscr{B}\left(\left(v_{q}-N a_{q}, v_{q}+N a_{q}\right)\right)}\right)\right) \\
& =\int_{B_{i_{1}} \times \cdots \times B_{i_{n}}} d\left(\bigotimes_{p=1}^{n} \frac{1}{2 N} \mathrm{Leb}\right) \\
& \quad \cdot \int_{\prod_{q \in I \backslash I_{n}} B_{q}} d\left(\left.\bigotimes_{q \in I \backslash I_{n}} \frac{1}{2 N} \mathrm{Leb}\right|_{\mathscr{B}\left(\left(v_{q}-N a_{q}, v_{q}+N a_{q}\right)\right)}\right) .
\end{aligned}
$$

Moreover, we have

$$
\begin{gathered}
\int_{\prod_{q \in I \backslash I_{n}} B_{q}} d\left(\left.\bigotimes_{q \in I \backslash I_{n}} \frac{1}{2 N} \operatorname{Leb}\right|_{\mathscr{B}\left(\left(v_{q}-N a_{q}, v_{q}+N a_{q}\right)\right)}\right) \\
=\int_{\prod_{q \in I \backslash I_{n}} B_{q}} d\left(\left.\bigotimes_{q \in I \backslash I_{n}} \frac{1}{2 N} \operatorname{Leb}\right|_{\mathscr{B}\left(B_{q}\right)}\right) \\
=\lim _{p \rightarrow+\infty} \int_{\prod_{q \in I_{p} \backslash I_{n}} B_{q}} d\left(\left.\bigotimes_{q \in I_{p} \backslash I_{n}} \frac{1}{2 N} \operatorname{Leb}\right|_{\mathscr{B}\left(B_{q}\right)}\right)
\end{gathered}
$$

(by Theorem 1)

$$
\begin{aligned}
= & \lim _{p \rightarrow+\infty} \int_{\prod_{q \in I_{p} \backslash I_{n}} g_{q}^{-1}\left(B_{q}\right)} \prod_{q \in I_{p} \backslash I_{n}}\left|\operatorname{det} J_{g_{q}}\left(x_{q}\right)\right| \\
& \cdot d\left(\left.\bigotimes_{q \in I_{p} \backslash I_{n}} \frac{1}{2 N} \operatorname{Leb}\right|_{\mathscr{B}\left(g_{q}^{-1}\left(B_{q}\right)\right)}\right)\left(x_{I_{p} \backslash I_{n}}\right)
\end{aligned}
$$

(by Proposition 38) 


$$
\begin{aligned}
= & \lim _{p \rightarrow+\infty} \frac{1}{\prod_{q \in I \backslash I_{p}}\left|\operatorname{det} J_{g_{q}}\left(x_{q}\right)\right|} \\
& \cdot \int_{\prod_{q \in I_{p} \backslash n} g_{q}^{-1}\left(B_{q}\right)} \prod_{q \in I \backslash I_{n}}\left|\operatorname{det} J_{g_{q}}\left(x_{q}\right)\right| \\
& \cdot d\left(\bigotimes_{q \in I_{p} \backslash I_{n}} \frac{1}{2 N} \operatorname{Leb} \mid\right. \\
= & \left.\int_{\prod_{q} \in I I_{q}} g_{q}^{-1}\left(B_{q}\right)\right) \\
g_{q}^{-1}\left(B_{q}\right) & \prod_{q \in I \backslash I_{n}}\left|\operatorname{det} J_{g_{q}}\left(x_{q}\right)\right|
\end{aligned}
$$

$$
\begin{aligned}
& \cdot d\left(\left.\bigotimes_{q \in I \backslash I_{n}} \frac{1}{2 N} \operatorname{Leb}\right|_{\mathscr{B}\left(g_{q}^{-1}\left(B_{q}\right)\right)}\right)\left(x_{I \backslash I_{n}}\right) \\
= & \int_{\prod_{q \in I I I_{n}} g_{q}^{-1}\left(B_{q}\right)} \prod_{q \in I \backslash I_{n}}\left|\operatorname{det} J_{g_{q}}\left(x_{q}\right)\right| \\
& \cdot d\left(\left.\bigotimes_{q \in I \backslash I_{n}} \frac{1}{2 N} \operatorname{Leb}\right|_{\mathscr{B}\left(\left(z_{q}-N b_{q}, z_{q}+N b_{q}\right)\right)}\right)\left(x_{I \backslash I_{n}}\right) .
\end{aligned}
$$

Then, from Proposition 39, formula (81) implies

$$
\begin{aligned}
\int_{B} d \lambda_{N, a, v}^{(k, I)=} & \int_{\left(\varphi^{(n, n)}\right)^{-1}\left(B_{i_{i}} \times \cdots \times B_{i_{n}}\right)}\left|\operatorname{det} J_{\varphi^{(n, n)}}\right| d\left(\bigotimes_{p=1}^{n} \frac{1}{2 N} \operatorname{Leb}\right) \cdot \int_{\prod_{q \in I I I_{n}} g_{q}^{-1}\left(B_{q}\right)} \prod_{q \in I \backslash I_{n}}\left|\operatorname{det} J_{g_{q}}\left(x_{q}\right)\right| \\
& \cdot d\left(\left.\bigotimes_{q \in I I_{n}} \frac{1}{2 N} \operatorname{Leb}\right|_{\mathscr{B}\left(\left(z_{q}-N b_{q}, z_{q}+N b_{q}\right)\right)}\right)\left(x_{I \backslash I_{n}}\right) \\
= & \int_{\left(\varphi^{(n)}\right)^{-1}(B)}\left|\operatorname{det} J_{\varphi^{(n)}}\right| d\left(\left(\bigotimes_{p=1}^{n} \frac{1}{2 N} \operatorname{Leb}\right) \otimes\left(\left.\bigotimes_{q \in I I_{n}} \frac{1}{2 N} \operatorname{Leb}\right|_{\mathscr{B}\left(\left(z_{q}-N b_{q}, z_{q}+N b_{q}\right)\right)}\right)\right) \\
= & \int_{\left(\varphi^{(n)}\right)^{-1}(B)}\left|\operatorname{det} J_{\varphi^{(n)}}\right| d\left(\left(\bigotimes_{p=1}^{k} \frac{1}{2 N} \operatorname{Leb}\right) \otimes\left(\left.\bigotimes_{q \in I I_{k}} \frac{1}{2 N} \operatorname{Leb}\right|_{\mathscr{B}\left(\left(z_{q}-N b_{q}, z_{q}+N b_{q}\right)\right)}\right)\right) \\
= & \int_{\left(\varphi^{(n)}\right)^{-1}(B)}\left|\operatorname{det} J_{\bar{\varphi}}\right| d \lambda_{N, b, z}^{(k, I)} .
\end{aligned}
$$

Consider the measures $\mu_{1}$ and $\mu_{2}$ on $\Sigma \equiv \mathscr{B}^{(I)}\left(E_{M_{1}, N, a, v}^{(k, I)}\right)$ defined by

$$
\begin{aligned}
& \mu_{1}(B)=\int_{B} d \lambda_{N, a, v}^{(k, I)}, \\
& \mu_{2}(B)=\int_{\left(\varphi^{(n)}\right)^{-1}(B)}\left|\operatorname{det} J_{\bar{\varphi}}\right| d \lambda_{N, b, z}^{(k, I)} ;
\end{aligned}
$$

from (83), $\mu_{1}$ and $\mu_{2}$ coincide on the set

$$
\mathscr{I}=\left\{B \in \Sigma: B=\prod_{i \in I} B_{i}\right\} ;
$$

moreover, we have $\mu_{1}\left(E_{M_{1}, N, a, v}^{(k, I)}\right)=\mu_{2}\left(E_{M_{1}, N, a, v}^{(k, I)}\right)<+\infty$, $E_{M_{1}, N, a, v}^{(k, I)} \in \mathscr{I}$, and so $\mu_{1}$ and $\mu_{2}$ are $\sigma$-finite on $\mathscr{I}$. Then, since $\mathscr{I}$ is a $\pi$-system on $E_{M_{1}, N, a, v}^{(k, I)}$ such that $\sigma(\mathscr{I})=\Sigma$, from Proposition $45, \forall B \in \mathscr{B}^{(I)}\left(E_{M_{1}, N, a, v}^{(k, I)}\right)$, we have

$$
\int_{B} d \lambda_{N, a, v}^{(k, I)}=\int_{\left(\varphi^{(n)}\right)^{-1}(B)}\left|\operatorname{det} J_{\bar{\varphi}}\right| d \lambda_{N, b, z}^{(k, I)}
$$

moreover, since $\left(\varphi^{(n)}\right)^{-1}(B) \subset E_{M_{2}, N, b, z}^{(k, I)}$ from Proposition 44, we have

$$
\begin{aligned}
& \int_{E_{M_{1}, N, a, v}^{(k, I)}} 1_{B} d \lambda_{N, a, v}^{(k, I)} \\
& \quad=\int_{E_{M_{2}, N,,, z, z}^{(k, I)}} 1_{B}\left(\varphi^{(n)}\right)\left|\operatorname{det} J_{\bar{\varphi}}\right| d \lambda_{N, b, z}^{(k, I)} .
\end{aligned}
$$

This implies that, if $\psi:\left(\mathbf{R}^{I}, \mathscr{B}^{(I)}\right) \rightarrow([0,+\infty), \mathscr{B}([0,+\infty)))$ is a simple function such that $\psi(x)=0, \forall x \notin E_{M_{1}, N, a, v}^{(k, I)}$, we have

$$
\int_{E_{M_{1}, N, a, v}^{(k, I)}} \psi d \lambda_{N, a, v}^{(k, I)}=\int_{E_{M_{2}, N, b, z}^{(k, I)}} \psi\left(\varphi^{(n)}\right)\left|\operatorname{det} J_{\bar{\varphi}}\right| d \lambda_{N, b, z}^{(k, I)}
$$

Then, if $l:\left(\mathbf{R}^{I}, \mathscr{B}^{(I)}\right) \rightarrow([0,+\infty), \mathscr{B}([0,+\infty)))$ is a measurable function such that $l(x)=0, \forall x \notin E_{M_{1}, N, a, v}^{(k, I)}$, and $\left\{\psi_{i}\right\}_{i \in \mathbf{N}}$ is a sequence of increasing positive simple functions 
over $\left(\mathbf{R}^{I}, \mathscr{B}^{(I)}\right)$ such that $\lim _{i \rightarrow+\infty} \psi_{i}=l, \psi_{i}(x)=0, \forall x \notin$ $E_{M_{1}, N, a, v}^{(k, I)}, \forall i \in \mathbf{N}$, from Beppo Levi theorem we have

$$
\begin{gathered}
\int_{E_{M_{1}, N, a, v}^{(k, I)}} l d \lambda_{N, a, v}^{(k, I)}=\lim _{i \rightarrow+\infty} \int_{E_{M_{1}, N, a, v}^{(k, I)}} \psi_{i} d \lambda_{N, a, v}^{(k, I)} \\
=\lim _{i \rightarrow+\infty} \int_{E_{M_{2}, N, b, z}^{(k, I)}} \psi_{i}\left(\varphi^{(n)}\right)\left|\operatorname{det} J_{\bar{\varphi}}\right| d \lambda_{N, b, z}^{(k, I)} \\
=\int_{E_{M_{2}, N, b, z}^{(k, I)}} l\left(\varphi^{(n)}\right)\left|\operatorname{det} J_{\bar{\varphi}}\right| d \lambda_{N, b, z}^{(k, I)} \\
=\lim _{n \rightarrow+\infty} \int_{E_{M_{2}, N, b, z}^{(k, I)}} l\left(\varphi^{(n)}\right)\left|\operatorname{det} J_{\bar{\varphi}}\right| d \lambda_{N, b, z}^{(k, I)} .
\end{gathered}
$$
form

In particular, formula (89) is true if $l$ is a function of the

$$
\begin{aligned}
& l(x)=l^{(m)}\left(x_{i_{1}}, \ldots, x_{i_{k}}\right) \prod_{j \in I \backslash I_{k}} 1_{B_{j}}\left(x_{j}\right), \\
& \forall x=\left(x_{j}: j \in I\right) \in \mathbf{R}^{I},
\end{aligned}
$$

where $l^{(k)}: \mathbf{R}^{k} \rightarrow[0,1]$ is a continuous function such that $l^{(k)}(\mathbf{x})=0, \forall \mathbf{x} \notin\left(-M_{1}, M_{1}\right)^{k}$, and $B_{j} \in \mathscr{B}\left(\left(v_{j}-N a_{j}, v_{j}+\right.\right.$ $\left.\left.N a_{j}\right)\right), \forall j \in I \backslash I_{k}$. In this case, let $\left\{f_{n}\right\}_{n \geq k}$ be the sequence of the measurable functions $f_{n}:\left(E_{M_{2}, N, b, z}^{(k, I)}, \mathscr{B}^{(I)}\left(E_{M_{2}, N, b, z}^{(k, I)}\right)\right) \rightarrow$ $([0,+\infty), \mathscr{B}([0,+\infty)))$ given by

$$
f_{n}(x)=l\left(\varphi^{(n)}(x)\right)\left|\operatorname{det} J_{\bar{\varphi}}(x)\right|,
$$

$$
\forall x \in E_{M_{2}, N, b, z}^{(k, I)}, \forall n \geq k ;
$$

$\forall n \geq k$, we have $\left|f_{n}\right| \leq g$, where $g:\left(E_{M_{2}, N, b, z}^{(k, I)}\right.$, $\left.\mathscr{B}^{(I)}\left(E_{M_{2}, N, b, z}^{(k, I)}\right)\right) \rightarrow([0,+\infty), \mathscr{B}([0,+\infty)))$ is the measurable function defined by

$$
g(x)=\left|\operatorname{det} J_{\bar{\varphi}}(x)\right|, \quad \forall x \in E_{M_{2}, N, b, z^{*}}^{(k, I)} .
$$

Moreover, we have

$$
\begin{array}{r}
g(x)=\left|\operatorname{det} J_{\varphi^{(m, m)}}\left(x_{i_{1}}, \ldots, x_{i_{k}}\right)\right| \prod_{j \in I \backslash I_{k}}\left|g_{j}^{\prime}\left(x_{j}\right)\right|, \\
\forall x \in E_{M_{2}, N, b, z}^{(k, I)}
\end{array}
$$

then, by definition of $E_{M_{2}, N, b, z}^{(k, I)}$ and since the functions $J_{\varphi^{(m, m)}}$ and $g_{j}^{\prime}, \forall j \in I \backslash I_{m}$, are continuous, there exists $\beta \in \mathbf{R}^{+}$such that $g(x) \leq \beta, \forall x \in E_{M_{2}, N, b, z}^{(k, I)}$, and so

$$
\begin{aligned}
& \int_{E_{M_{2}, N, b, z}^{(k, I)}} g d \lambda_{N, b, z}^{(k, I)} \leq \beta \lambda_{N, b, z}^{(k, I)}\left(E_{M_{2}, N, b, z}^{(k, I)}\right)=\beta \prod_{p \in I_{k}} \frac{1}{2 N} \\
& \cdot \operatorname{Leb}\left(\left(-M_{2}, M_{2}\right)\right) \\
& \cdot \prod_{q \in I \backslash I_{k}} \frac{1}{2 N} \operatorname{Leb}\left(\left(z_{q}-N b_{q}, z_{q}+N b_{q}\right)\right)=\frac{\beta M_{2}^{k}}{N^{k}} \\
& \cdot \prod_{q \in I \backslash I_{k}} b_{q}<+\infty .
\end{aligned}
$$

Moreover, we have $\lim _{n \rightarrow+\infty} \varphi^{(n)}=\varphi$, and so $\lim _{n \rightarrow+\infty} f_{n}(x)=$ $l(\varphi(x))\left|\operatorname{det} J_{\bar{\varphi}}(x)\right|, \forall x \in E_{M_{2}, N, b, z}^{(k, I)}$; then, from the dominated convergence theorem:

$$
\begin{gathered}
\lim _{n \rightarrow+\infty} \int_{E_{M_{2}, N, b, z}^{(k, I)}} l\left(\varphi^{(n)}\right)\left|\operatorname{det} J_{\bar{\varphi}}\right| d \lambda_{N, b, z}^{(k, I)} \\
=\int_{E_{M_{2}, N, b, z}^{(k, I)}} l(\varphi)\left|\operatorname{det} J_{\bar{\varphi}}\right| d \lambda_{N, b, z}^{(k, I)} ;
\end{gathered}
$$

consequently, from (89), we have

$$
\int_{E_{M_{1}, N, a, v}^{(k, I)}} l d \lambda_{N, a, v}^{(k, I)}=\int_{E_{M_{2}, N, b, z}^{(k, I)}} l(\varphi)\left|\operatorname{det} J_{\bar{\varphi}}\right| d \lambda_{N, b, z}^{(k, I)} .
$$

Let $B=\prod_{j \in I} B_{j} \in \mathscr{B}^{(I)}\left(E_{M_{1}, N, a, v}^{(k, I)}\right)$, where $B_{j}=\left(a_{j}, b_{j}\right)$, $\forall j \in I_{k}$; moreover, $\forall n \in \mathbf{N}^{*}$, consider the function $l_{n}: \mathbf{R}^{I} \rightarrow$ $[0,1]$ defined by

$$
\begin{aligned}
l_{n}(x)=l_{n}^{(k)}\left(x_{j}: j \in I_{k}\right) \prod_{j \in I \backslash I_{k}} 1_{B_{j}}\left(x_{j}\right), & \\
& \forall x=\left(x_{j}: j \in I\right) \in \mathbf{R}^{I},
\end{aligned}
$$

where

$l_{n}^{(k)}(\mathbf{x})$

$$
= \begin{cases}1 & \text { if } \mathbf{x} \in \prod_{j \in I_{k}}\left(a_{j}+\frac{\delta_{j}}{n}, b_{j}-\frac{\delta_{j}}{n}\right) \\ \frac{\left\|\mathbf{x}-\mathbf{x}_{2}\right\|_{I_{m}}}{\left\|\mathbf{x}_{1}-\mathbf{x}_{2}\right\|_{I_{m}}} & \text { if } \mathbf{x} \in\left(\prod_{j \in I_{k}} B_{j}\right) \backslash \prod_{j \in I_{k}}\left(a_{j}+\frac{\delta_{j}}{n}, b_{j}-\frac{\delta_{j}}{n}\right) \\ 0 & \text { if } \mathbf{x} \notin \prod_{j \in I_{k}} B_{j},\end{cases}
$$

$\delta_{j}=\left(b_{j}-a_{j}\right) / 2, \forall j \in I_{k}, \mathbf{x}=\left(x_{j}: j \in I_{k}\right), \mathbf{x}_{1}=r \cap$ $\partial\left(\prod_{j \in I_{k}}\left(a_{j}+\delta_{j} / n, b_{j}-\delta_{j} / n\right)\right), \mathbf{x}_{2}=r \cap \partial\left(\prod_{j \in I_{k}} B_{j}\right)$, where $r$ is the half-line with initial point $\prod_{j \in I_{k}}\left(\left(a_{j}+b_{j}\right) / 2\right)$ and containing $\mathbf{x}$. Since $\left\{l_{n}\right\}_{n \in \mathbf{N}^{*}}$ is an increasing positive sequence such that $\lim _{n \rightarrow+\infty} l_{n}=1_{B}$, and since the function $l_{n}^{(k)}: \mathbf{R}^{m} \rightarrow[0,1]$ is continuous, from Beppo Levi theorem and (96), we have

$$
\begin{gathered}
\int_{E_{M_{1}, N, a, v}^{(k, I)}} 1_{B} d \lambda_{N, a, v}^{(k, I)}=\lim _{n \rightarrow+\infty} \int_{E_{M_{1}, N, a, v}^{(k, I)}} l_{n} d \lambda_{N, a, v}^{(k, I)} \\
=\lim _{n \rightarrow+\infty} \int_{E_{M_{2}, N, b, z}^{(k, I)}} l_{n}(\varphi)\left|\operatorname{det} J_{\bar{\varphi}}\right| d \lambda_{N, b, z}^{(k, I)} \\
=\int_{E_{M_{2}, N, b, z}^{(k, I)}} 1_{B}(\varphi)\left|\operatorname{det} J_{\bar{\varphi}}\right| d \lambda_{N, b, z^{*}}^{(k, I)}
\end{gathered}
$$

Moreover, Proposition 45 implies that formula (99) is true $\forall B \in \mathscr{B}^{(I)}\left(E_{M_{1}, N, a, v}^{(k, I)}\right)$. Consider the measures $\mu$ and $v$ on $\left(E_{N, a, v}^{(k, I)}, \mathscr{B}^{(I)}\left(E_{N, a, v}^{(k, I)}\right)\right)$ defined by

$$
\begin{aligned}
& \mu(B)=\int_{B} d \lambda_{N, a, v}^{(k, I)}, \\
& v(B)=\int_{\varphi^{-1}(B)}\left|\operatorname{det} J_{\bar{\varphi}}\right| d \lambda_{N, b, z}^{(k, I)},
\end{aligned}
$$


and set $B_{n}=B \cap E_{n, N, a, v}^{(k, I)}, \forall n \in \mathbf{N}^{*}$ and $\forall B \in \mathscr{B}^{(I)}\left(E_{N, a, v}^{(k, I)}\right)$. Since $B_{n} \subset B_{n+1}, A^{-1}\left(B_{n}\right) \subset A^{-1}\left(B_{n+1}\right), \bigcup_{n \in \mathbf{N}^{*}} B_{n}=B$ and $\bigcup_{n \in \mathbf{N}^{*}} A^{-1}\left(B_{n}\right)=A^{-1}(B)$, from the continuity property of $\mu$ and $v$ and (99), we have

$$
\begin{aligned}
\int_{B} d \lambda_{N, a, v}^{(k, I)} & =\lim _{n \rightarrow+\infty} \int_{B_{n}} d \lambda_{N, a, v}^{(k, I)} \\
& =\lim _{n \rightarrow+\infty} \int_{\varphi^{-1}\left(B_{n}\right)}\left|\operatorname{det} J_{\bar{\varphi}}\right| d \lambda_{N, b, z}^{(k, I)} \\
& =\int_{\varphi^{-1}(B)}\left|\operatorname{det} J_{\bar{\varphi}}\right| d \lambda_{N, b, z}^{(k, I)} .
\end{aligned}
$$

Then, suppose that $B \in \mathscr{B}^{(I)}$; from Lemma 46, formula (101), and Proposition 44, we have

$$
\begin{aligned}
\int_{\mathbf{R}^{I}} 1_{B} d \lambda_{N, a, v}^{(k, I)} & =\int_{B \cap E_{N, a, v}^{(k, I)}} d \lambda_{N, a, v}^{(k, I)} \\
& =\int_{\varphi^{-1}\left(B \cap E_{N, a, v}^{(k, I)}\right)}\left|\operatorname{det} J_{\bar{\varphi}}\right| d \lambda_{N, b, z}^{(k, I)} \\
& =\int_{\varphi^{-1}(B) \cap E_{N, b, z}^{(k, I)}}\left|\operatorname{det} J_{\bar{\varphi}}\right| d \lambda_{N, b, z}^{(k, I)} \\
& =\int_{\mathbf{R}^{I}} 1_{B}(\varphi)\left|\operatorname{det} J_{\bar{\varphi}}\right| d \lambda_{N, b, z}^{(k, I)} ;
\end{aligned}
$$

thus, by proceeding as in the proof of formula (89), for any measurable function $f:\left(\mathbf{R}^{I}, \mathscr{B}^{(I)}\right) \rightarrow$ $([0,+\infty), \mathscr{B}([0,+\infty)))$, we obtain

$$
\int_{\mathbf{R}^{I}} f d \lambda_{N, a, v}^{(k, I)}=\int_{\mathbf{R}^{I}} f(\varphi)\left|\operatorname{det} J_{\bar{\varphi}}\right| d \lambda_{N, b, z}^{(k, I)} .
$$

Then, for any measurable function $f:\left(\mathbf{R}^{I}, \mathscr{B}^{(I)}\right) \rightarrow(\mathbf{R}, \mathscr{B})$ such that $f^{+}\left(\right.$or $\left.f^{-}\right)$is $\lambda_{N, a, v}^{(k, I)}$-integrable and for any $B \in \mathscr{B}^{(I)}$, we have

$$
\begin{aligned}
\int_{B} f d \lambda_{N, a, v}^{(k, I)}= & \int_{\mathbf{R}^{I}} f^{+} 1_{B} d \lambda_{N, a, v}^{(k, I)}-\int_{\mathbf{R}^{I}} f^{-} 1_{B} d \lambda_{N, a, v}^{(k, I)} \\
= & \int_{\mathbf{R}^{I}}\left(f^{+} 1_{B}\right)(\varphi)\left|\operatorname{det} J_{\bar{\varphi}}\right| d \lambda_{N, b, z}^{(k, I)} \\
& -\int_{\mathbf{R}^{I}}\left(f^{-} 1_{B}\right)(\varphi)\left|\operatorname{det} J_{\bar{\varphi}}\right| d \lambda_{N, b, z}^{(k, I)} \\
= & \int_{\mathbf{R}^{I}}\left(f 1_{B}\right)(\varphi)\left|\operatorname{det} J_{\bar{\varphi}}\right| d \lambda_{N, b, z}^{(k, I)} \\
= & \int_{\varphi^{-1}(B)} f(\varphi)\left|\operatorname{det} J_{\bar{\varphi}}\right| d \lambda_{N, b, z}^{(k, I)} .
\end{aligned}
$$

\section{Problems for Further Study}

A natural extension of this paper is the generalization of Theorem 47 , by substituting the $(m, \sigma)$-standard functions for more general functions $\varphi: U \subset E_{I} \rightarrow E_{I}$ such that, for any $i \in I \backslash I_{m}$, the function $\varphi_{i}: U \subset E_{I} \rightarrow \mathbf{R}$ depends on a finite number of variables.

Moreover, a natural application of this paper, in the probabilistic framework, is the development of the theory of the infinite-dimensional continuous random elements, defined in the paper [1]. In particular, we can prove the formula of the density of such random elements composed with the $(m, \sigma)$-standard functions given in the change of variables' formula in Theorem 47. Consequently, it is possible to introduce many random elements that generalize the wellknown continuous random vectors in $\mathbf{R}^{m}$ (e.g., the Gaussian random elements in $E_{I}$ defined by the $(m, \sigma)$-standard matrices) and to develop some theoretical results and some applications in the statistical inference. It is possible also to define a convolution between the laws of two independent and infinite-dimensional continuous random elements, as in the finite case.

Furthermore, we can generalize paper [11] by considering the recursion $\left\{X_{n}\right\}_{n \in \mathbf{N}}$ on $[0, p)^{\mathbf{N}^{*}}$ defined by

$$
X_{n+1}=A X_{n}+B_{n} \quad(\bmod p) \text {, }
$$

where $X_{0}=x_{0} \in E_{I}, A$ is a biunique, linear, integer and ( $m, \sigma)$-standard function, $p \in \mathbf{R}^{+}$, and $\left\{B_{n}\right\}_{n \in \mathbf{N}}$ is a sequence of independent and identically distributed random elements on $E_{I}$. Our target is to prove that, with some assumptions on the law of $B_{n}$, the sequence $\left\{X_{n}\right\}_{n \in \mathbf{N}}$ converges with geometric rate to a random element with law $\bigotimes_{i \in \mathbf{N}^{*}}\left(\left.(1 / p) \operatorname{Leb}\right|_{\mathscr{B}([0, p))}\right)$. Moreover, we wish to quantify the rate of convergence in terms of $A, p, m$, and the law of $B_{n}$, and to prove that, if $A$ has an eigenvalue that is a root of 1 , then $O\left(p^{2}\right)$ steps are necessary to achieve randomness.

\section{Competing Interests}

The author declares that there is no conflict of interests regarding the publication of this paper.

\section{References}

[1] C. Asci, "Integration over an infinite-dimensional Banach space and probabilistic applications," International Journal of Analysis, vol. 2014, Article ID 404186, 10 pages, 2014.

[2] R. Léandre, "Lebesgue measure in infinite dimension as an infinite-dimensional distribution," Journal of Mathematical Sciences, vol. 159, no. 6, pp. 833-836, 2009.

[3] N. Tsilevich, A. Vershik, and M. Yor, "An infinite-dimensional analogue of the Lebesgue measure and distinguished properties of the gamma process," Journal of Functional Analysis, vol. 185, no. 1, pp. 274-296, 2001.

[4] R. Baker, "Lebesgue measure' on $R^{\infty}$," Proceedings of the American Mathematical Society, vol. 113, pp. 1023-1029, 1991.

[5] L. Accardi, O. G. Smolyanov, and M. O. Smolyanova, "Change of variable formulas for infinite-dimensional distributions," Mathematical Notes, vol. 60, pp. 212-215, 1996.

[6] M. M. Rao, Measure Theory and Integration, Wiley-Interscience, New York, NY, USA, 1987.

[7] J. Weidmann, Linear Operators in Hilbert Spaces, Springer, New York, NY, USA, 1980. 
[8] S. Lang, Linear Algebra, Springer, New York, NY, USA, 3rd edition, 1987.

[9] S. Lang, Analysis II, Addison-Wesley, Reading, Mass, USA, 1969.

[10] P. Billingsley, Probability and Measure, Wiley-Interscience, New York, NY, USA, 3rd edition, 1995.

[11] C. Asci, "Convergence in total variation of an affine random recursion in $[0, p)^{k}$ to a uniform random vector," Markov Processes and Related Fields, vol. 19, no. 1, pp. 125-140, 2013. 


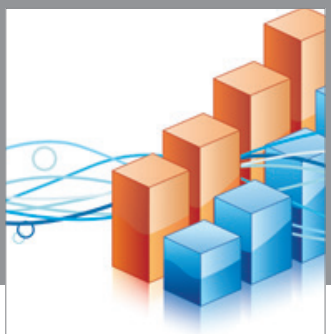

Advances in

Operations Research

vatem alat4

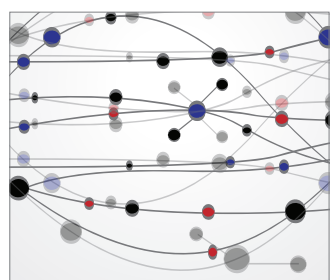

\section{The Scientific} World Journal
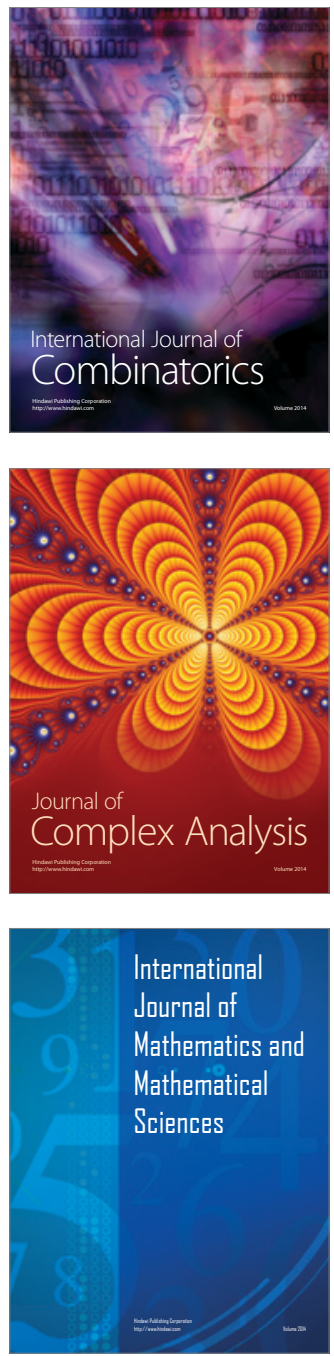
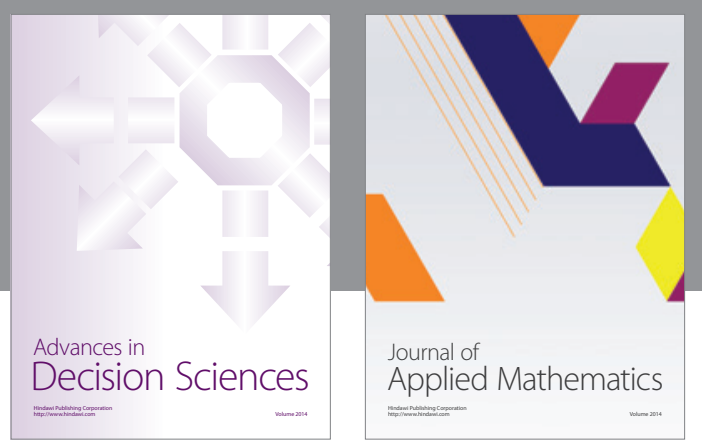

Algebra

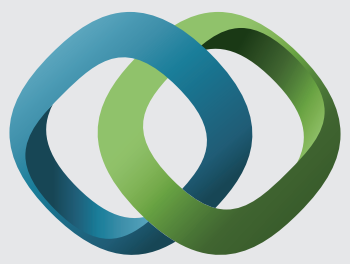

\section{Hindawi}

Submit your manuscripts at

http://www.hindawi.com
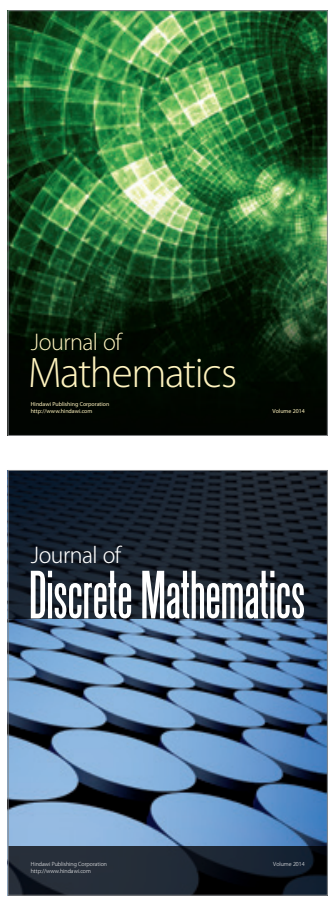

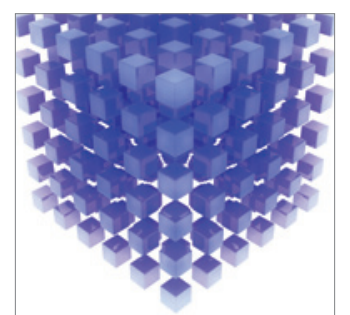

Mathematical Problems in Engineering
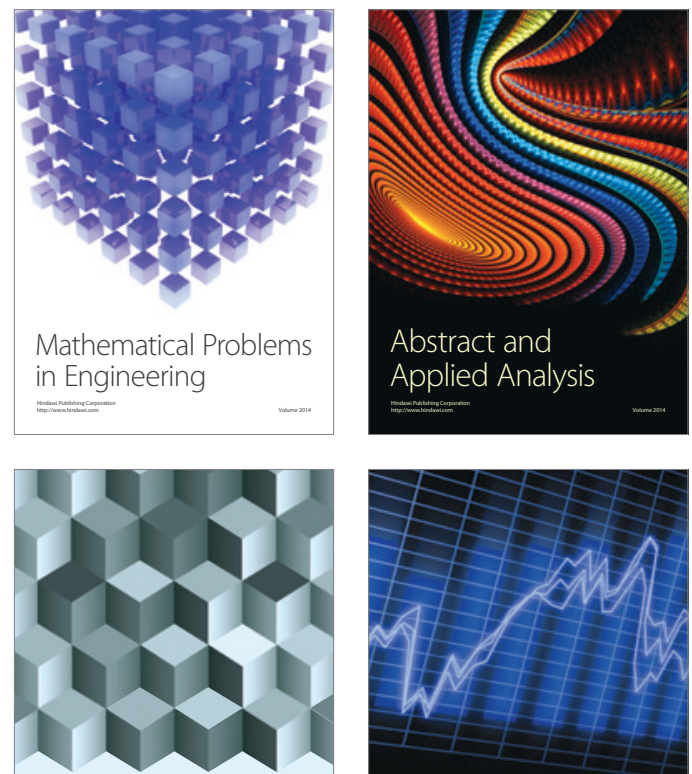

Journal of

Function Spaces

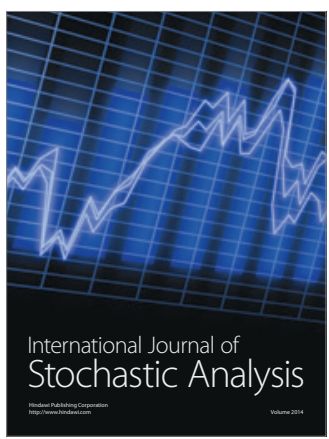

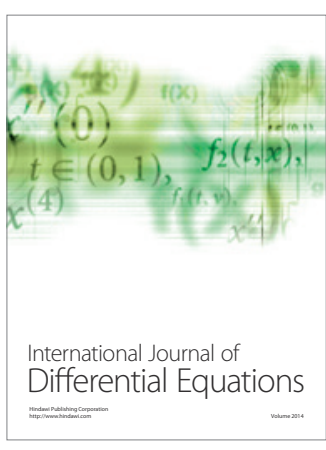
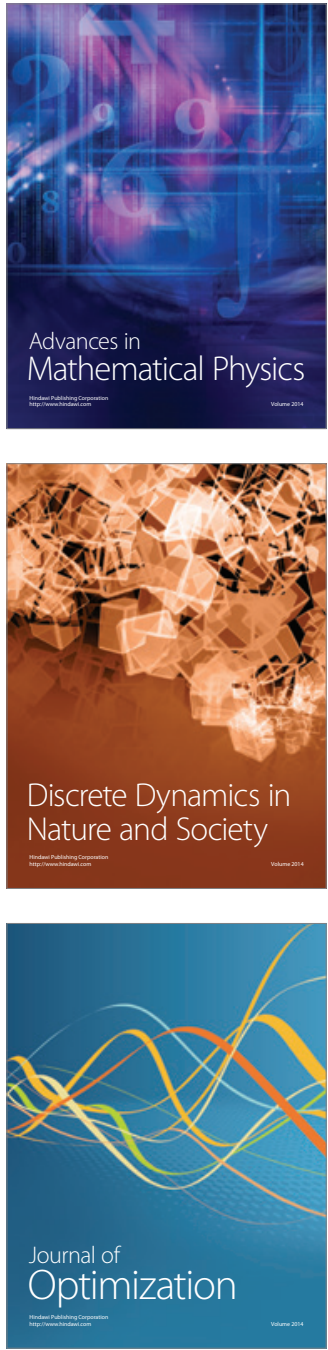